\title{
The low-luminosity Type II SN 2016aqf: a well-monitored spectral evolution of the $\mathrm{Ni} / \mathrm{Fe}$ abundance ratio
}

Tomás E. Müller-Bravo ${ }^{\circledR},{ }^{1 \star}$ Claudia P. Gutiérrez ${ }^{\circledR}, 1$ Mark Sullivan ${ }^{\circledR}, 1$ Anders Jerkstrand, ${ }^{2,3}$ Joseph P. Anderson, ${ }^{4}$ Santiago González-Gaitán ${ }^{\circledR}, 5$ Jesper Sollerman, ${ }^{2}$ Iair Arcavi, ${ }^{6,7}$ Jamison Burke, ${ }^{8,9}$ Lluís Galbany ${ }^{\circledR},{ }^{10}$ Avishay Gal-Yam, ${ }^{11}$ Mariusz Gromadzki, ${ }^{12}$ Daichi Hiramatsu, ${ }^{8,9}$ Griffin Hosseinzadeh, ${ }^{13}$ D. Andrew Howell, ${ }^{8,9}$ Cosimo Inserra ${ }^{\circledR},{ }^{14}$ Erki Kankare, ${ }^{15}$ Alexandra Kozyreva ${ }^{\circledR},{ }^{3}$ Curtis McCully, ${ }^{8}$ Matt Nicholl ${ }^{\circledR},{ }^{16,17}$ Stephen Smartt, ${ }^{18}$ Stefano Valenti ${ }^{19}$ and Dave R. Young ${ }^{18}$

Affiliations are listed at the end of the paper

Accepted 2020 June 29. Received 2020 June 26; in original form 2020 April 14

\begin{abstract}
Low-luminosity Type II supernovae (LL SNe II) make up the low explosion energy end of core-collapse SNe, but their study and physical understanding remain limited. We present SN 2016aqf, an LL SN II with extensive spectral and photometric coverage. We measure a $V$-band peak magnitude of $-14.58 \mathrm{mag}$, a plateau duration of $\sim 100 \mathrm{~d}$, and an inferred ${ }^{56} \mathrm{Ni}$ mass of $0.008 \pm 0.002 \mathrm{M}_{\odot}$. The peak bolometric luminosity, $\mathrm{L}_{\mathrm{bol}} \approx 10^{41.4} \mathrm{erg} \mathrm{s}^{-1}$, and its spectral evolution are typical of other $\mathrm{SNe}$ in the class. Using our late-time spectra, we measure the [O I] $\lambda \lambda 6300,6364$ lines, which we compare against SN II spectral synthesis models to constrain the progenitor zero-age main-sequence mass. We find this to be $12 \pm 3 \mathrm{M}_{\odot}$. Our extensive late-time spectral coverage of the $[\mathrm{Fe}$ II] $\lambda 7155$ and [Ni II] $\lambda 7378$ lines permits a measurement of the $\mathrm{Ni} / \mathrm{Fe}$ abundance ratio, a parameter sensitive to the inner progenitor structure and explosion mechanism dynamics. We measure a constant abundance ratio evolution of $0.081_{-0.010}^{+0.009}$ and argue that the best epochs to measure the ratio are at $\sim 200-300 \mathrm{~d}$ after explosion. We place this measurement in the context of a large sample of SNe II and compare against various physical, light-curve, and spectral parameters, in search of trends that might allow indirect ways of constraining this ratio. We do not find correlations predicted by theoretical models; however, this may be the result of the exact choice of parameters and explosion mechanism in the models, the simplicity of them, and/or primordial contamination in the measured abundance ratio.
\end{abstract}

Key words: techniques: photometric-spectroscopic telescopes - supernovae: individual: SN 2016aqf - transients: supernovae.

\section{INTRODUCTION}

Massive stars of $M \gtrsim 8 \mathrm{M}_{\odot}$ finish their lives with the collapse of their iron core, which releases great amounts of energy and produces explosions known as core collapse supernovae (CCSNe). These explosions can leave behind compact remnants in the form of neutron stars or black holes, although the exact details of the outcomes are not well understood. Within the different classes of CCSNe, Type II SNe (SNe II), characterized by the presence of hydrogen in their spectra, are the most common (Li et al. 2011; Shivvers et al. 2017). SNe II are a heterogeneous class, with light curves showing different decline rates across a continuum (e.g. Anderson et al. 2014) from plateau (SNe IIP; with a pseudo-constant luminosity for $\sim 70-120 \mathrm{~d}$ ) to linear decliners (SNe IIL, or fastdeclining $\mathrm{SNe}$ ). The light curves generally show two distinct phases: an optically thick phase, driven by a combination of the expansion of the ejecta (which pushes the photosphere outwards) and the recombination of hydrogen (which pushes the photosphere inwards),

^E-mail: t.e.muller-bravo@soton.ac.uk and a later optically thin phase, powered by the radioactive decay of ${ }^{56} \mathrm{Co}$.

$\mathrm{SNe}$ II show a large diversity in luminosities, with peak $V$-band maximum absolute magnitudes ranging from $\sim-13.5$ to $\sim-19$ mag, and an average of about $-16.7 \mathrm{mag}(\sigma=1.01 \mathrm{mag}$; Anderson et al. 2014). Several low-luminosity SNe II (LL SNe II), generally events with $V \gtrsim-16$ mag (e.g. Kulkarni \& Kasliwal 2009; Smartt et al. 2015, see also Gal-Yam 2017; however, note that Pastorello 2012 proposes an alternative definition), have been found in the past decades (e.g. Turatto et al. 1998; Pastorello 2012; Spiro et al. 2014; Lisakov et al. 2018).

The prototype of this faint sub-class is SN 1997D (de Mello, Benetti \& Massone 1997; Turatto et al. 1998). SN 1997D displayed a low-luminosity and low-expansion velocity. However, it was discovered several weeks after peak, with no well-constrained explosion epoch. The first statistical study of this sub-class was that of Pastorello et al. (2004), who found the class to be characterized by narrow spectral lines (P-Cygni profiles) and low expansion velocities (a few $1000 \mathrm{~km} \mathrm{~s}^{-1}$ during the late photospheric phase), suggesting low explosion energies $\left(E_{\exp } \lesssim\right.$ few times $10^{50} \mathrm{erg}$ ). Their bolometric luminosity during the recombination ranges between $\sim 10^{41} \mathrm{erg} \mathrm{s}^{-1}$ 
and $\sim 10^{42} \mathrm{erg} \mathrm{s}^{-1}$, with SN 1999br (Pastorello et al. 2004) and SN 2010id (Gal-Yam et al. 2011) being the faintest SNe II discovered. They also show lower exponential decay luminosity than the bulk of SNe II, which reflects their low ${ }^{56} \mathrm{Ni}$ masses $\left(\mathrm{M}_{\mathrm{Ni}} \lesssim 10^{-2} \mathrm{M}_{\odot}\right)$, in agreement with the low explosion energies as expected from the $\mathrm{M}_{\mathrm{Ni}}-$ $E_{\text {exp }}$ relation found in different studies (e.g. Kushnir 2015; Pejcha \& Prieto 2015; Müller et al. 2017). Spiro et al. (2014) have since expanded the statistical study of LL SNe II, adding several objects and finding similar characteristics to those found by Pastorello et al. (2004). While the current sample of nebular spectra of LL SNe II is growing, the study of additional events with better cadence and higher signal-to-noise data is essential for understanding their observed diversity.

The progenitors of LL SNe II have been shown to be red supergiants (RSGs) with relatively small Zero Age Main Sequence (ZAMS) masses $\left(M \lesssim 15 \mathrm{M}_{\odot}\right)$ using archival pre-SN imaging (e.g. Smartt et al. 2009; Smartt 2015) and hydrodynamical models (e.g. Dessart et al. 2013b; Martinez \& Bersten 2019). However, other studies have suggested the possibility that their progenitors are more massive RSGs with large amounts of fallback material (e.g. Zampieri et al. 2003). Theoretical studies have shown that the nebular [OI] $\lambda \lambda 6300,6364$ doublet is a good tracer of the progenitor core mass, and, therefore, of the progenitor ZAMS mass (e.g. Jerkstrand et al. 2012, 2014, 2018, hereafter J12, J14, and J18; and some other studies as well, e.g. Lisakov et al. 2017, 2018), making the late-time spectral evolution extremely important for the study of SN progenitors. Furthermore, nebular nucleosynthesis diagnosis is so far consistent with the lack of massive progenitors above $\sim 20 \mathrm{M}_{\odot}$ (e.g. J14, Jerkstrand et al. 2015a; Valenti et al. 2016).

In addition to the study of the nebular [O I] $\lambda \lambda 6300,6364$ doublet as progenitor mass estimator, the $\mathrm{Ni} / \mathrm{Fe}$ abundance ratio, measured from the [Fe II] $\lambda 7155$ and [Ni II] $\lambda 7378$ lines, have been shown to be important for the understanding of the inner structure of the progenitor and the explosion mechanism dynamics, as the observed iron-group yields are linked to the temperature, density, and neutron excess of the layers that become fuel for the rapid burning process of the explosion (Jerkstrand et al. 2015a,b, hereafter J15a, J15b). However, there are few studies of this ratio, mainly due to the lack of late-time spectra and the absence of these features in the available data in the literature.

In this paper, we study SN 2016aqf: a well-observed (i.e. excellent spectral and photometric coverage) LL SN II, discovered soon after explosion, with $M_{V}^{\max }=-14.58 \mathrm{mag}$, a plateau duration of $\sim 100 \mathrm{~d}$, and a measured $\mathrm{M}_{\mathrm{Ni}}=0.0010 \mathrm{M}_{\odot}$ (see Sections 3.2 and 4.1). The nebular spectra show the [O I] $\lambda \lambda 6300,6364$ doublet. The He I $\lambda 7065$ emission line is also seen in the spectra of SN2016aqf, a line associated to $\mathrm{SNe}$ with a low progenitor mass, but not present in every LL SN II and not well understood. In addition, SN 2016aqf is one of the few cases where the $[\mathrm{Fe} \mathrm{II}] \lambda 7155$ and $[\mathrm{Ni}$ II] $\lambda 7393$ lines (produced by ${ }^{56} \mathrm{Ni}$ and ${ }^{58} \mathrm{Ni}$, respectively) can be seen in the nebular spectra $(\sim 150-330 \mathrm{~d}$ after the explosion $)$ over $\sim 170 \mathrm{~d}$. This extended coverage of the $\mathrm{Ni} / \mathrm{Fe}$ abundance ratio presents a unique opportunity to study its evolution and serves as a test for current late-time spectral modelling as well as providing a rich legacy data set.

This paper is structured as follows: in Section 2.1, we describe the observations, data reduction, and host galaxy of SN 2016aqf. In Section 3, we show the light curve, colour, and spectral evolution of SN 2016aqf and compare it with other LL SNe II. In Section 4.2, we estimate the physical parameters of SN 2016aqf, while in Section 5, we discuss our findings. Finally, our conclusions are in Section 6.
Throughout this paper, we assume a flat $\Lambda \mathrm{CDM}$ cosmology with $H_{0}=70 \mathrm{~km} \mathrm{~s}^{-1} \mathrm{Mpc}^{-1}, \Omega_{\mathrm{M}}=0.3$, and $\Omega_{\Lambda}=0.7$, as these values are widely used in the literature (e.g. Gutiérrez et al. 2018) and the $H_{0}$ value lies between the value measured from the Cosmic Microwave Background (CMB) (Planck Collaboration XIII, 2016) and local measurements (e.g. Riess et al. 2018).

\section{OBSERVATIONS, REDUCTIONS, AND HOST GA LA XY}

\subsection{SN photometry and spectroscopy}

SN 2016aqf (ASASSN-16cc) was discovered on 2016 February 26, at 04:33:36 UTC (57444.19 MJD) by the All-Sky Automated Survey for Supernovae $^{1}$ (ASAS-SN; Shappee et al. 2014) at RA $=05 \mathrm{~h} 46 \mathrm{~m} 23^{\mathrm{s}} .91$ and Dec. $=-52^{\circ} 05^{\prime} 18^{\prime \prime} .9$, in NGC 2101 (Brown et al. 2016) at $z=0.004016$ (Lauberts \& Valentijn 1989). On 2016 February 27, SN 2016aqf was classified as an SN II (Hosseinzadeh et al. 2016; Jha \& Miszalski 2016). Based on the low luminosity of the host $\left(M_{\mathrm{B}}=-17.66 \mathrm{mag}\right.$ as in Gutiérrez et al. 2018, although see Section 2.2), we commenced a follow-up campaign with the extended Public ESO Spectroscopic Survey of Transient Objects (ePESSTO) as part of the programme 'SNe II in Low-luminosity host galaxies'.

The final pre-explosion non-detection in the $V$ band, reported $3 \mathrm{~d}$ before the date of classification by ASAS-SN (57442 MJD), has a limiting magnitude of $\sim 16.7 \mathrm{mag}$, which does not give a stringent constraint on the explosion epoch. Previous non-detections have similar limiting magnitudes. Hence, we decided to estimate the explosion epoch using the spectral matching technique (e.g. Anderson et al. 2014; Gutiérrez et al. 2017). We used GELATO ${ }^{2}$ (Harutyunyan et al. 2008) to find good spectral matches to the highest resolution spectrum of SN 2016aqf, as it is also one of the first spectra taken (57446 MJD, see below). From the best matching templates, we calculated a mean epoch of the spectrum of $\sim 6 \mathrm{~d}$ after explosion and a mean error added with the standard deviation of the explosion epochs in quadrature of $\sim 4 \mathrm{~d}$. This gives an explosion epoch of MJD $57440.19 \pm 4$ (slightly different to the estimated epoch in Gutiérrez et al. 2018, as they used the non-detection).

Optical BVgri imaging of SN 2016aqf was obtained with the 1.0-m telescope network of the Las Cumbres Observatory (LCO; Brown et al. 2013) as part of both ePESSTO and the 'Las Cumbres Observatory SN Key Project', with data taken from 8 to $311 \mathrm{~d}$ after explosion. All photometric data were reduced following the prescriptions described by Firth et al. (2015). This pipeline subtracts a deep reference image constructed using data obtained in the $B V g r i$ bands $3 \mathrm{yr}$ after the first detection of SN 2016aqf to remove the hostgalaxy light using a point-spread-function (PSF) matching routine. $\mathrm{SN}$ photometry is then measured from the difference images using a PSF-fitting technique. Fig. 1 shows the SN position within the host galaxy. The photometry of SN2016aqf is presented in Table 1 .

Spectroscopic observations were obtained with the ESO Faint Object Spectrograph and Camera version 2 (EFOSC2; Buzzoni et al. 1984) at the 3.58-m ESO New Technology Telescope (NTT), the FLOYDS spectrograph (Brown et al. 2013) on the Faulkes Telescope South (FTS), and the Robert Stobie Spectrograph (RSS; Burgh et al. 2003; Kobulnicky et al. 2003) at the Southern African Large Telescope (SALT). FLOYDS spectra were taken as part of the 'Las Cumbres Observatory SN Key Project'. The observations

\footnotetext{
${ }^{1}$ http://www.astronomy.ohio-state.edu/assassin/index.shtml

${ }^{2}$ https://gelato.tng.iac.es/gelato/
} 


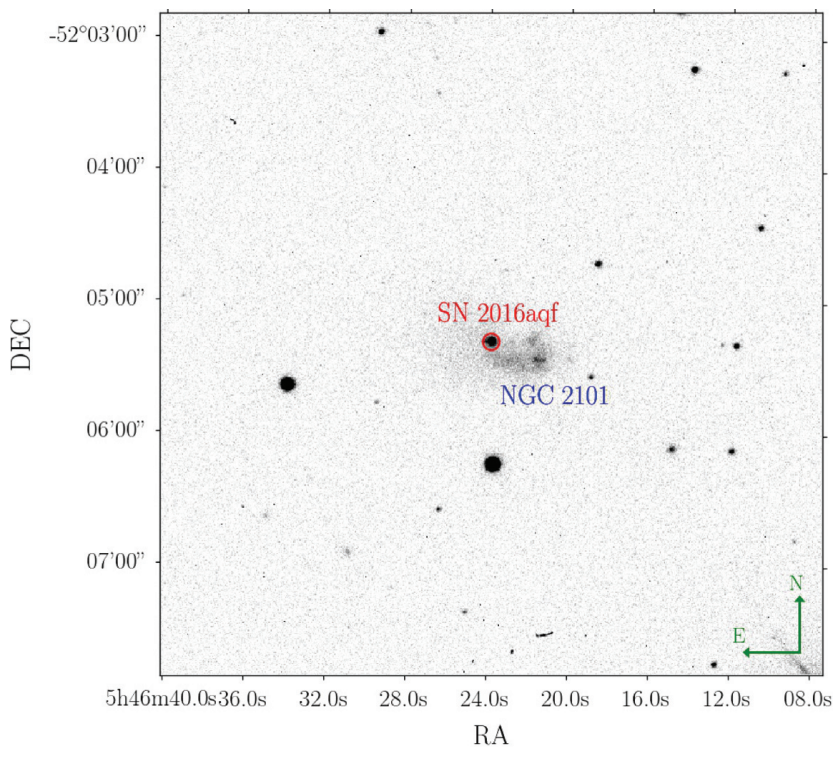

Figure 1. $r$-band image of NGC 2101 with SN 2016aqf marked. Data from the 1.0-m Las Cumbres Observatory telescopes (MJD $=57514,74 \mathrm{~d}$ after explosion).

include phases from 2 to $348 \mathrm{~d}$ after explosion. EFOSC2 spectra, obtained with grism \#13, cover 3500-9300 A at a $21.2 \AA$ resolution, the FLOYDS spectra have wavelength coverage of $\sim 3200-10000 \AA$ with a resolution of $\sim 18 \AA$, and the RSS spectrum (Jha \& Miszalski 2016) covers $3600-9200 \AA$ at $\sim 7 \AA$ resolution. The data reduction of the EFOSC2 spectra was performed using the PESSTO pipeline ${ }^{3}$ (Smartt et al. 2015), while the FLOYDS data were reduced using the PYRAF-based FLOYDSSPEC pipeline ${ }^{4}$ (Valenti et al. 2014). All spectra are available via the WISeREP ${ }^{5}$ repository (Yaron \& Gal-Yam 2012). Spectral information is summarized in Table 2.

\subsection{Host galaxy}

Photometry of NGC 2101 was obtained with the LCO 1.0-m telescope network, and spectroscopy with VLT/FORS2, around 3 yr after the SN explosion (2019 February 6, at 04:38:48 UTC). We estimated a galaxy distance of $\mu=30.16 \pm 0.27$ mag (see Section 3.2), consistent with the Tully-Fisher value of $\mu=$ $30.61 \pm 0.80 \mathrm{mag}$, as reported in the NASA/IPAC Extragalactic Database $^{6}$ (NED). Adopting the distance estimated in this work, the galaxy has $M_{\mathrm{B}}=-17.22 \pm 0.34 \mathrm{mag}$, which is consistent with the value reported in Gutiérrez et al. (2018, $-17.66 \mathrm{mag}$ ), given the large uncertainties from the reported distance. We use the total apparent corrected $B$-magnitude, with the total $B$-magnitude error as reported in HyperLEDA, using error propagation. The radial velocity corrected for Local Group infall on to Virgo is $883 \pm 3 \mathrm{~km} \mathrm{~s}^{-1}$ (Theureau et al. 1998; Terry, Paturel \& Ekholm 2002), as reported in HyperLEDA, a value that we use to estimate the corrected redshift of SN 2016aqf.

From the spectrum of the $\mathrm{H}$ II region at the position of the $\mathrm{SN}$, we measure the emission line fluxes of $\mathrm{H} \alpha, \mathrm{H} \beta,[\mathrm{N} \mathrm{II}]$, and [O III]. We estimate the star formation rate (SFR) from the $\mathrm{H} \alpha$ line

\footnotetext{
${ }^{3}$ https://github.com/svalenti/pessto

${ }^{4}$ https://github.com/svalenti/FLOYDS_pipeline

${ }^{5}$ https://wiserep.weizmann.ac.il/

${ }^{6} \mathrm{http}: / /$ ned.ipac.caltech.edu/
}

as $\mathrm{SFR}=2.3 \pm 0.6 \times 10^{-1} \mathrm{M}_{\odot} \mathrm{yr}^{-1}$ using the calibration from Kennicutt \& Evans (2012), where the uncertainty is driven by the uncertainty in the distance. Using the calibration of Marino et al. (2013), we then estimate a gas-phase metallicity of $(12+\log (\mathrm{O} / \mathrm{H}))_{\mathrm{O} 3 \mathrm{~N} 2}=$ $8.144 \pm 0.025$ dex and $(12+\log (\mathrm{O} / \mathrm{H}))_{\mathrm{N} 2}=8.134 \pm 0.042$ dex, i.e. below the solar value of 8.69 dex (Asplund et al. 2009). This is low compared to many other SN II host galaxies but not uncommon (e.g. Anderson et al. 2016). However, the metallicity does not follow the relation found with the Fe II $\lambda 5018$ pEW (e.g. Dessart et al. 2014; Anderson et al. 2016; Gutiérrez et al. 2018). This may be caused by the lower temperatures in LL SNe II, which causes the earlier appearance of the Fe II lines in these objects (Gutiérrez et al. 2017).

\section{RESULTS AND ANALYSIS}

\subsection{Extinction corrections}

We adopt a Milky Way extinction value of $E(B-V)_{\mathrm{MW}}=0.047 \mathrm{mag}$ and correct our photometry using the prescription of Schlafly \& Finkbeiner (2011) and the Cardelli, Clayton \& Mathis (1989) reddening law with $R_{V}=3.1$. To estimate the host galaxy extinction, we investigated the equivalent width (EW) of the NaID ( $\lambda \lambda 5889$, 5895) absorption, a well-known tracer of gas, metals, and dust (e.g. Richmond et al. 1994; Munari \& Zwitter 1997; Turatto, Benetti \& Cappellaro 2003; Poznanski, Prochaska \& Bloom 2012). We note that these relations tend to have large uncertainties.

The spectrum at $+6 \mathrm{~d}$ is the only one that seems to shows $\mathrm{Na}$ I D absorption lines from the MW and the host galaxy. We used the relations for one line $\left(D_{1}\right)$ and two lines $\left(D_{1}+D_{2}\right)$ from Poznanski et al. (2012), obtaining upper limits of $E(B-V) \lesssim 0.028 \pm 0.011 \mathrm{mag}$ and $E(B-V) \lesssim 0.032 \pm 0.006 \mathrm{mag}$, respectively. This gives a weighted average value of $E(B-V) \lesssim 0.031 \mathrm{mag}$. Given this very small level of extinction (and its uncertainty), we choose not to make an extinction correction to the $\mathrm{SN}$ data. We do not use other methods to estimate this value as they rely on the SN colour; de Jaeger et al. (2018) showed that the majority of colour dispersion of SNe II is intrinsic to the SN.

\subsection{Light curve and distance}

The BVgri-band light curves of SN 2016aqf (Fig. 2) cover 8-311 d after explosion (all phases in this paper are relative to the estimated explosion epoch). As the host galaxy is not in the Hubble flow, we estimated the distance to SN 2016aqf using the Standardized Candle Method (Hamuy \& Pinto 2002), which relates the velocity of the ejecta of an SN II to its luminosity during the plateau, and the relation of Kasen \& Woosley (2009, equation 17) for a redshiftindependent distance estimate. We calculate the distance modulus $\mu=30.16 \pm 0.27 \mathrm{mag}(10.8 \pm 1.4 \mathrm{Mpc})$, which gives $M_{V}^{\max }=$ -14.58 mag and a mid-plateau $V$-band luminosity of -14.63 mag (note the plateau luminosity is slightly brighter; $M_{V}^{\max }$ represents the maximum luminosity from the peak closest to the bolometric peak). We estimated $M_{V}^{\max }$ from the first epoch of photometry, given that the last non-detection helps to obtain a good constrain.

During the recombination phase, the SN shows an increase in the Vri-bands luminosity, probably due to its low temperature, which shifts the peak luminosity from the ultraviolet (UV) to redder bands more rapidly compared to normal SNe II. The gap in observations between 80 and $150 \mathrm{~d}$ was caused by the SN going behind the sun and coincides with the $\mathrm{SN}$ transitioning from the optically thick to the optically thin phase. The $V$ band decreases by $\sim 2$ mag across the gap in the light curve and is an estimate of the decrease caused by the 
Table 1. SN 2016aqf BVgri-band photometry between +5 and +311 d. $B V$ bands are in Vega magnitude system, while gri bands are in $\mathrm{AB}$ magnitude system.

\begin{tabular}{|c|c|c|c|c|c|c|c|c|c|c|c|}
\hline MJD & Phase & $B$ & $\sigma_{B}$ & $V$ & $\sigma_{V}$ & $g$ & $\sigma_{g}$ & $r$ & $\sigma_{r}$ & $i$ & $\sigma_{i}$ \\
\hline 57448 & 8 & 15.851 & 0.006 & 15.851 & 0.006 & 15.851 & 0.006 & 15.851 & 0.006 & 15.851 & 0.006 \\
\hline 57452 & 12 & 15.985 & 0.005 & 15.985 & 0.005 & 15.985 & 0.005 & 15.985 & 0.005 & 15.985 & 0.005 \\
\hline 57455 & 15 & 16.057 & 0.010 & 16.057 & 0.010 & 16.057 & 0.010 & 16.057 & 0.010 & 16.057 & 0.010 \\
\hline 57456 & 16 & 16.045 & 0.034 & 16.045 & 0.034 & 16.045 & 0.034 & 16.045 & 0.034 & 16.045 & 0.034 \\
\hline 57457 & 17 & 16.033 & 0.103 & 16.033 & 0.103 & - & - & - & - & - & - \\
\hline 57458 & 18 & 16.095 & 0.039 & 16.095 & 0.039 & 16.095 & 0.039 & 16.095 & 0.039 & - & - \\
\hline 57459 & 19 & 16.131 & 0.008 & 16.131 & 0.008 & 16.131 & 0.008 & 16.131 & 0.008 & 16.131 & 0.008 \\
\hline 57460 & 20 & 16.190 & 0.005 & 16.190 & 0.005 & 16.190 & 0.005 & 16.190 & 0.005 & 16.190 & 0.005 \\
\hline 57462 & 22 & 16.268 & 0.031 & 16.268 & 0.031 & 16.268 & 0.031 & 16.268 & 0.031 & 16.268 & 0.031 \\
\hline 57468 & 28 & 16.459 & 0.009 & 16.459 & 0.009 & 16.459 & 0.009 & 16.459 & 0.009 & 16.459 & 0.009 \\
\hline 57474 & 34 & 16.534 & 0.010 & 16.534 & 0.010 & 16.534 & 0.010 & 16.534 & 0.010 & 16.534 & 0.010 \\
\hline 57480 & 40 & 16.576 & 0.008 & 16.576 & 0.008 & 16.576 & 0.008 & 16.576 & 0.008 & 16.576 & 0.008 \\
\hline 57487 & 47 & 16.650 & 0.009 & 16.650 & 0.009 & 16.650 & 0.009 & 16.650 & 0.009 & 16.650 & 0.009 \\
\hline 57493 & 53 & 16.754 & 0.015 & 16.754 & 0.015 & 16.754 & 0.015 & 16.754 & 0.015 & 16.754 & 0.015 \\
\hline 57499 & 59 & 16.812 & 0.014 & 16.812 & 0.014 & 16.812 & 0.014 & 16.812 & 0.014 & 16.812 & 0.014 \\
\hline 57505 & 65 & 16.824 & 0.015 & 16.824 & 0.015 & 16.824 & 0.015 & 16.824 & 0.015 & 16.824 & 0.015 \\
\hline 57512 & 72 & 16.890 & 0.017 & 16.890 & 0.017 & - & - & 16.890 & 0.017 & 16.890 & 0.017 \\
\hline 57514 & 74 & 16.912 & 0.012 & 16.912 & 0.012 & 16.912 & 0.012 & 16.912 & 0.012 & 16.912 & 0.012 \\
\hline 57523 & 83 & - & - & - & - & 16.258 & 0.007 & 16.258 & 0.007 & - & - \\
\hline 57524 & 84 & - & - & - & - & 16.289 & 0.008 & 16.289 & 0.008 & - & - \\
\hline 57526 & 86 & 17.182 & 0.030 & 17.182 & 0.030 & 17.182 & 0.030 & 17.182 & 0.030 & 17.182 & 0.030 \\
\hline 57598 & 158 & 19.108 & 0.034 & 19.108 & 0.034 & 19.108 & 0.034 & 19.108 & 0.034 & 19.108 & 0.034 \\
\hline 57617 & 177 & 19.216 & 0.095 & 19.216 & 0.095 & 19.216 & 0.095 & 19.216 & 0.095 & 19.216 & 0.095 \\
\hline 57684 & 244 & 20.311 & 0.041 & 20.311 & 0.041 & 20.311 & 0.041 & 20.311 & 0.041 & 20.311 & 0.041 \\
\hline 57704 & 264 & 20.430 & 0.074 & 20.430 & 0.074 & 20.430 & 0.074 & 20.430 & 0.074 & 20.430 & 0.074 \\
\hline 57726 & 286 & - & - & 19.546 & 0.077 & 19.546 & 0.077 & - & - & - & - \\
\hline 57727 & 287 & - & - & - & - & 19.795 & 0.049 & 19.795 & 0.049 & 19.795 & 0.049 \\
\hline 57728 & 288 & 20.709 & 0.063 & 20.709 & 0.063 & 20.709 & 0.063 & 20.709 & 0.063 & 20.709 & 0.063 \\
\hline 57749 & 309 & 20.925 & 0.120 & 20.925 & 0.120 & 20.925 & 0.120 & 20.925 & 0.120 & 20.925 & 0.120 \\
\hline 57750 & 310 & 20.717 & 0.073 & 20.717 & 0.073 & 20.717 & 0.073 & 20.717 & 0.073 & 20.717 & 0.073 \\
\hline 57751 & 311 & 20.932 & 0.076 & 20.932 & 0.076 & 20.932 & 0.076 & 20.932 & 0.076 & 20.932 & 0.076 \\
\hline
\end{tabular}

Table 2. The UTC dates mark the beginning of the exposures. Phase with respect to the explosion epoch (MJD 57440.19).

\begin{tabular}{lccccc}
\hline UTC date & MJD & Phase & Range $(\AA)$ & Resolution $(\AA)$ & Telescope/instrument \\
\hline 2016-02-27T09:54:44.475 & 57445.4 & 5 & $3250-9300$ & 18.0 & FTS/FLOYDS-S \\
2016-02-27T21:00:25.837 & 57445.9 & 6 & $3600-9200$ & 7.0 & SALT/RSS \\
2016-03-01T11:24:32.205 & 57448.5 & 8 & $3250-10000$ & 18.0 & FTS/FLOYDS-S \\
2016-03-06T10:09:18.022 & 57453.4 & 13 & $3300-10001$ & 18.0 & FTS/FLOYDS-S \\
2016-03-09T04:39:49.731 & 57456.2 & 16 & $3640-9235$ & 21.2 & NTT/EFOSC2 \\
2016-03-10T12:46:20.372 & 57457.5 & 17 & $3299-10000$ & 18.0 & FTS/FLOYDS-S \\
2016-03-15T10:13:25.851 & 57462.4 & 22 & $3250-10000$ & 18.0 & FTS/FLOYDS-S \\
2016-03-22T11:10:18.554 & 57469.5 & 29 & $3900-9999$ & 18.0 & FTS/FLOYDS-S \\
2016-03-30T09:29:37.393 & 57477.4 & 37 & $3401-10000$ & 18.0 & FTS/FLOYDS-S \\
2016-04-06T08:58:10.460 & 57484.4 & 44 & $3299-9999$ & 18.0 & FTS/FLOYDS-S \\
2016-04-13T10:06:55.453 & 57491.4 & 51 & $3599-10000$ & 18.0 & FTS/FLOYDS-S \\
2016-04-15T08:39:52.609 & 57493.4 & 53 & $3600-10000$ & 18.0 & FTS/FLOYDS-S \\
2016-04-16T00:26:16.687 & 57494.1 & 54 & $3645-9239$ & 21.2 & NTT/EFOSC2 \\
2016-04-22T08:55:30.146 & 57500.4 & 60 & $3950-10000$ & 18.0 & FTS/FLOYDS-S \\
2016-05-04T09:27:45.944 & 57512.4 & 72 & $3650-10000$ & 18.0 & FTS/FLOYDS-S \\
2016-07-26T09:49:26.448 & 57595.4 & 155 & $3645-9239$ & 21.2 & NTT/EFOSC2 \\
2016-08-08T09:39:55.699 & 57608.4 & 168 & $3639-9233$ & 21.2 & NTT/EFOSC2 \\
2016-09-11T08:20:03.866 & 57642.3 & 202 & $3640-9233$ & 21.2 & NTT/EFOSC2 \\
2016-09-29T07:23:09.743 & 57660.3 & 220 & $3636-9232$ & 21.2 & NTT/EFOSC2 \\
2016-11-07T07:57:09.799 & 57699.3 & 259 & $3639-9232$ & 21.2 & NTT/EFOSC2 \\
2016-11-19T04:31:19.658 & 57711.2 & 271 & $3636-9231$ & 21.2 & NTT/EFOSC2 \\
2016-12-03T06:56:26.427 & 57725.3 & 285 & $3639-9232$ & 21.2 & NTT/EFOSC2 \\
2016-12-21T05:55:04.628 & 57743.2 & 303 & $3640-9233$ & 21.2 & NTT/EFOSC2 \\
2017-01-17T02:55:39.708 & 57770.1 & 330 & $3639-9233$ & 21.2 & NTT/EFOSC2 \\
2017-02-07T02:46:40.051 & 57791.1 & 351 & $3640-9233$ & 21.2 & NTT/EFOSC2 \\
\hline
\end{tabular}




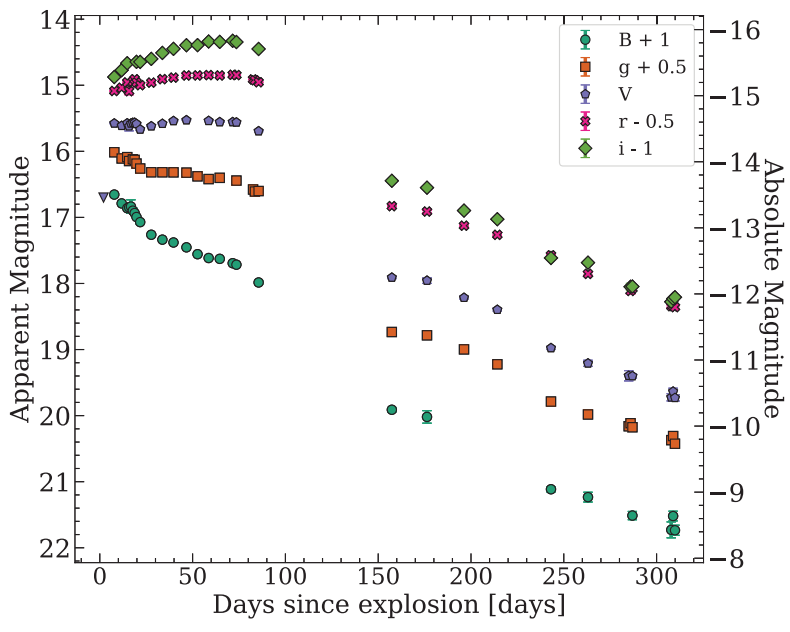

Figure 2. SN 2016aqf $B V g r i$-band photometry from +8 to +311 d. $B V$ bands are in Vega magnitude system, while gri bands are in AB magnitude system. The last non-detection in $V$ band is also shown (inverted triangle). The SN was not visible around the transition from the optically thick to the optically thin phase. Offsets have been applied to the photometry for visualization purposes. As in all figures in this paper, the photometry is corrected for MW extinction but not host extinction, and the data are in the rest frame.

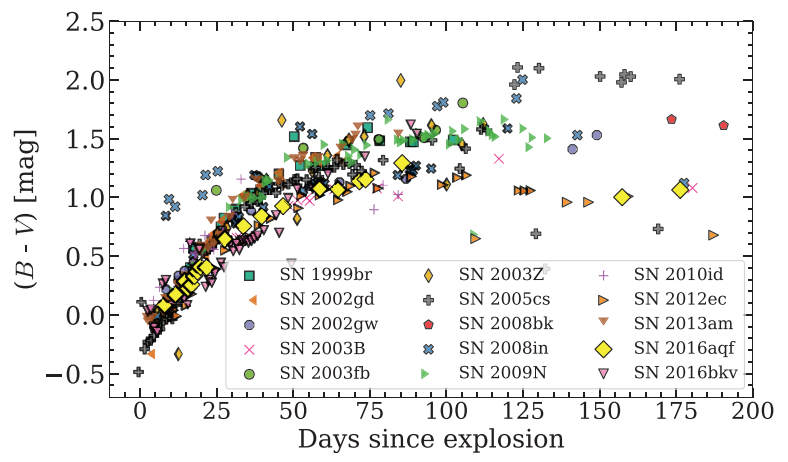

Figure 3. $(B-V)$ colour evolution of SN 2016aqf compared to our sample of LL SNe II. All data are corrected for MW and host galaxy extinction (except for those SNe with host extinction values reported as upper limits in Table 3.) Notice that the dispersion generally increases with time. Uncertainties are not shown as they are relatively small in general.

transition from plateau to nebular phase, smaller than other LL SNe II ( 3-5 mag; e.g. Spiro et al. 2014). We measured the decline rate in the $V$ band at early epochs $\left(t \lesssim 20 \mathrm{~d} ; s_{1}\right)$, in the plateau $\left(s_{2}\right)$, and in the exponential decay tail $\left(s_{3}\right)$ as defined in Anderson et al. (2014, see Section 4.2 for the $t_{\mathrm{pt}}$ used), obtaining $s 1=0.65_{-0.12}^{+0.13} \mathrm{mag} 100 \mathrm{~d}^{-1}$, $s 2=-0.08_{-0.01}^{+0.01} \operatorname{mag} 100 \mathrm{~d}^{-1}$, and $s 3=1.22_{-0.02}^{+0.02} \mathrm{mag} 100 \mathrm{~d}^{-1} \cdot M_{\text {tail }}$ was not measured as the early decline of the exponential decay tail was not observed.

\subsection{Colour evolution}

In Fig. 3, we show the $(B-V)$ colour curve (corrected for MW extinction) of SN 2016aqf during the first $200 \mathrm{~d}$. At the beginning of the observations ( $8 \mathrm{~d}$ ), it has a colour close to $0 \mathrm{mag}$, which slowly increases to around 1.0 mag at $\sim+50 \mathrm{~d}$ and $\sim 1.3$ mag before the gap in coverage.

For comparison, we form a sample of other LL SNe II from the literature with good data coverage and similar properties to our ob- ject: SN 1999br (Hamuy 2003; Pastorello et al. 2004; Gutiérrez et al. 2017), SN 2002gd, SN 2002gw, SN 2003B, SN 2003fb, SN 2003Z, SN 2004fx, SN 2005cs, SN 2008bk, SN 2008in, SN 2009N, SN2010id, SN 2013am, and SN2016bkv. These SNe and their references are in Table 3. In addition, we include SN 2012ec (Maund et al. 2013), a non-LL SN II, as a reference as it has a well-measured $\mathrm{Ni} / \mathrm{Fe}$ abundance ratio, used in our later analysis. For this comparison sample, we use photometry and spectra obtained from the 'Open Supernova Catalog' (Guillochon et al. 2017) and WISeREP (Yaron \& Gal-Yam 2012). Note that we used only epochs with both $B$ and $V$ photometry to calculate colour, without applying interpolations. The photometry of this sample is corrected for MW extinction (see Section 3.1), and host galaxy extinction, using the values from the references in Table 3. However, we do not correct for host galaxy extinction when the reported value is an upper limit (this does not represent a problem, given the relatively small extinction values, $\mathrm{A}_{V}$ $<0.1 \mathrm{mag})$.

The $(B-V)$ evolution of SN 2016aqf is in general flatter than the bulk of our sample, showing similar colours at early epochs $(t \lesssim$ $15 \mathrm{~d})$, but becoming slightly bluer at later epochs $(t \gtrsim 25 \mathrm{~d})$, similar to SN 2012ec. After $\sim 100 \mathrm{~d}$, the dispersion in the colour evolution of our sample starts increasing, probably due to the faintness of these objects.

\subsection{Bolometric light curve}

We estimated the bolometric light curve of SN 2016aqf by applying the bolometric correction from Lyman, Bersier \& James (2014) (assuming a cooling phase of $20 \mathrm{~d})$. We use the $(g-i)$ colour as it shows the smallest dispersion. Most SNe in our LL SN II sample have only $B V R I$ data, so, to be consistent, we calculated their bolometric light curves (correcting for MW extinction only) by applying the relation from Lyman et al. (2014) as well, but with $(B-I)$ colour as it has the smallest dispersion within the available bands, using the distances from Table 3. Only epochs with simultaneous $B$ and $I$ bands (or $g$ and $i$ for SN 2016aqf) were used. The light curves are shown in Fig. 4 (SN 2008bk is not shown as it does not have epochs with simultaneous $B$ and $I$ coverage). Unfortunately, as the relations from Lyman et al. (2014) work only in a given colour range, we cannot estimate the bolometric light curve during the nebular phase of some of the SNe.

The luminosity of SN 2016aqf at peak is $L_{\mathrm{bol}} \approx 10^{41.4} \mathrm{erg} \mathrm{s}^{-1}$, estimated from the first epoch with photometry. The luminosity of SN 2016aqf during the cooling phase generally decreases less steeply than other LL SNe II. During the plateau phase, the luminosity falls to $L_{\mathrm{bol}} \approx 10^{41.3} \mathrm{erg} \mathrm{s}^{-1}$, placing it in the mid-luminosity range of our sample (between SN 2005cs and SN 2002gd). After the gap, the SN has a luminosity of $L_{\text {bol }} \approx 10^{40.5} \mathrm{erg} \mathrm{s}^{-1}$, dropping to $L_{\text {bol }} \approx$ $10^{39.7} \mathrm{erg} \mathrm{s}^{-1}$ at $+300 \mathrm{~d}$. The exponential tail is steeper than ${ }^{56} \mathrm{Co}$ decay (0.98 mag per 100 d Woosley, Pinto \& Hartmann 1989), although shallower than the decay in the $V$ band, presumably due to $\gamma$-ray leakage.

\subsection{Early spectral evolution}

The spectra of SN 2016aqf have narrower lines than spectra of normal SNe II, suggesting low expansion velocities and low explosion energies. Spectra obtained during the optically thick phase are shown in Fig. 5. During the first 2 weeks, the evolution is mainly dominated by a blue continuum and Balmer lines, showing P-Cygni profiles of $\mathrm{H} \alpha$ and $\mathrm{H} \beta$. Fe II $\lambda 4924, \lambda 5018, \lambda 5169$ and $\mathrm{Ca}$ II $\lambda \lambda \lambda 8498,8542$, 
Table 3. SN II sample used throughout this work. The data for this sample were taken from the references cited in column References.

\begin{tabular}{|c|c|c|c|c|c|c|c|c|c|c|}
\hline SN & $\mathrm{z}$ & $\begin{array}{l}\mathrm{M}_{\mathrm{Ni}} \\
\left(\mathrm{M}_{\odot}\right)\end{array}$ & $\begin{array}{c}\sigma_{\mathrm{Ni}}^{-} \\
\left(\mathrm{M}_{\odot}\right)\end{array}$ & $\begin{array}{c}\sigma_{\mathrm{Ni}}^{+} \\
\left(\mathrm{M}_{\odot}\right)\end{array}$ & $\begin{array}{c}\mu \\
(\mathrm{mag})\end{array}$ & $\begin{array}{c}\sigma_{\mu} \\
(\mathrm{mag})\end{array}$ & $\begin{array}{c}A_{V}(\mathrm{MW}) \\
(\mathrm{mag})\end{array}$ & $\begin{array}{l}A_{V}(\mathrm{Host}) \\
\quad(\mathrm{mag})\end{array}$ & Host & References \\
\hline SN1997D & 0.004059 & 0.005 & 0.004 & 0.004 & 30.74 & 0.92 & 0.057 & $\lesssim 0.060$ & NGC 1536 & $\begin{array}{l}\text { Turatto et al. (1998), Zampieri et al. (2003) } \\
\text { Spiro et al. (2014) }\end{array}$ \\
\hline SN1999br & 0.00323 & 0.002 & 0.001 & 0.001 & 30.97 & 0.83 & 0.063 & 0.000 & NGC 4900 & $\begin{array}{l}\text { Hamuy (2003), Pastorello et al. (2004), } \\
\text { Gutiérrez et al. (2017) }\end{array}$ \\
\hline SN2002gd & 0.00892 & $<0.003$ & - & - & 32.87 & 0.35 & 0.178 & 0.000 & NGC 7537 & Spiro et al. (2014), Gutiérrez et al. (2017) \\
\hline SN2002gw & 0.01028 & 0.012 & 0.004 & 0.003 & 32.98 & 0.23 & 0.051 & 0.000 & NGC 922 & $\begin{array}{l}\text { Anderson et al. (2014), Galbany et al. (2016), } \\
\text { Gutiérrez et al. (2017) }\end{array}$ \\
\hline SN2003B & 0.00424 & 0.017 & 0.009 & 0.006 & 31.11 & 0.28 & 0.072 & 0.180 & NGC 1097 & $\begin{array}{l}\text { Blondin et al. (2006), Anderson et al. (2014), } \\
\text { Galbany et al. (2016), Gutiérrez et al. (2017) }\end{array}$ \\
\hline SN2003fb & 0.01754 & $>0.017$ & - & - & 34.43 & 0.12 & 0.482 & - & UGC 11522 & $\begin{array}{l}\text { Papenkova et al. (2003), Anderson et al. (2014), } \\
\text { Gutiérrez et al. (2017) }\end{array}$ \\
\hline SN2003Z & 0.0043 & 0.005 & 0.003 & 0.003 & 31.70 & 0.60 & 0.104 & 0.000 & NGC 2742 & Utrobin, Chugai \& Pastorello (2007), Spiro et al. (2014) \\
\hline SN2004fx & 0.00892 & 0.014 & 0.006 & 0.004 & 32.82 & 0.24 & 0.274 & 0.000 & MCG -02-14-003 & $\begin{array}{l}\text { Park \& Li (2004), Anderson et al. (2014), } \\
\text { Gutiérrez et al. (2017) }\end{array}$ \\
\hline SN2005cs & 0.002 & 0.006 & 0.003 & 0.003 & 29.46 & 0.60 & 0.095 & 0.171 & M 51 & $\begin{array}{l}\text { Pastorello et al. (2006), Pastorello et al. (2009), } \\
\text { Spiro et al. (2014) }\end{array}$ \\
\hline SN2007aa & 0.004887 & 0.032 & 0.009 & 0.009 & 31.95 & 0.27 & 0.070 & 0.000 & NGC 4030 & $\begin{array}{l}\text { Anderson et al. (2014), Gutiérrez et al. (2017), } \\
\text { This Work }\end{array}$ \\
\hline SN2008bk & 0.000767 & 0.007 & 0.001 & 0.001 & 27.68 & 0.13 & 0.052 & 0.000 & NGC 7793 & $\begin{array}{l}\text { Van Dyk et al. (2012), Anderson et al. (2014), } \\
\text { Spiro et al. (2014), Gutiérrez et al. (2017) }\end{array}$ \\
\hline SN2008in & 0.005224 & 0.012 & 0.005 & 0.005 & 30.60 & 0.20 & 0.060 & 0.080 & NGC 4303 & $\begin{array}{l}\text { Roy et al. (2011), Anderson et al. (2014), } \\
\text { Gutiérrez et al. (2017) }\end{array}$ \\
\hline SN2009N & 0.003456 & 0.020 & 0.004 & 0.004 & 31.67 & 0.11 & 0.056 & 0.100 & NGC 4487 & $\begin{array}{l}\text { Takáts et al. (2014), Anderson et al. (2014), } \\
\text { Spiro et al. (2014), Gutiérrez et al. (2017) }\end{array}$ \\
\hline SN2010id & 0.01648 & - & - & - & 32.86 & 0.50 & 0.162 & 0.167 & NGC 7483 & Gal-Yam et al. (2011), Spiro et al. (2014) \\
\hline SN2012A & 0.0025 & 0.011 & 0.004 & 0.004 & 29.96 & 0.15 & 0.085 & $\sim 0.010$ & NGC 3239 & Tomasella et al. (2013), J15a \\
\hline SN2012aw & 0.0026 & 0.060 & 0.010 & 0.010 & 29.97 & 0.03 & 0.074 & 0.143 & NGC 3351 & Fraser et al. (2012), Bose et al. (2013), J14, J15a \\
\hline SN2012ec & 0.00469 & 0.040 & 0.015 & 0.015 & 31.19 & 0.13 & 0.071 & 0.372 & NGC 1084 & Barbarino et al. (2015), J15a \\
\hline SN2013am & 0.002692 & 0.015 & 0.006 & 0.011 & 30.54 & 0.40 & 0.066 & 1.705 & NGC 3623 & Zhang et al. (2014), Tomasella et al. (2018) \\
\hline SN2016aqf & 0.004016 & 0.008 & 0.002 & 0.002 & 30.16 & 0.27 & 0.146 & $\lesssim 0.096$ & NGC 2101 & This Work \\
\hline SN2016bkv & 0.002 & 0.0216 & 0.0014 & 0.0014 & 30.79 & 0.05 & 0.045 & $\lesssim 0.016$ & NGC 3184 & Nakaoka et al. (2018), Hosseinzadeh et al. (2018) \\
\hline
\end{tabular}

8662 then appear, becoming prominent at later epochs. The Na ID appears at around 1 month. Sc II/Fe II $\lambda 5531$, Sc II $\lambda 5663, \lambda 6247$, and Ba II $\lambda 6142$ appear at around $+50 \mathrm{~d}$. O I $\lambda 7774$ is weakly present after 1 month.

Fig. 6 shows the spectra of SN 2016aqf with other SNe from our comparison sample. The Fe II lines are present in all SNe, although in SN 2016aqf, they are generally weaker. SN 2016aqf is similar to SN 2002gw and SN 2010id, with a relatively featureless spectrum between $\mathrm{H} \beta$ and $\mathrm{H} \alpha$. However, we see no major differences with the rest of the sample at $\sim+15 \mathrm{~d}$.

At around $+50 \mathrm{~d}$ (Fig. 6), SN 2016aqf resembles SN 2009N, with the difference that the Sc II/Fe II $\lambda 5531$, Sc II $\lambda 5663, \lambda 6247$, and Ba II $\lambda 6142$ lines are weaker (and weaker than most other $\mathrm{SNe}$ in our sample). O I $\lambda 7774$ is seen in the spectrum of most SNe, except SN 2002gd and SN 2016bkv where the signal-to-noise/resolution of the spectra precludes a secure identification. Most $\mathrm{SNe}$ have very similar Fe II and Ca II NIR line profiles. SN 2016aqf does not display any other peculiarity with respect to the comparison sample. Note that host galaxy extinction may be substantial for SN 2013am (Zhang et al. 2014; Tomasella et al. 2018), explaining the drop in flux at the bluer end of this SN.

Table 4 shows a list of lines with pseudo-Equivalent Width (pEW, not corrected for instrumental resolution), including the full width at half maximum (FWHM, not corrected for instrumental resolution) of $\mathrm{H} \alpha$, measured from the spectra of SN 2016aqf during the optically thick phase.

\subsection{Nebular spectral evolution}

Fig. 7 shows the spectra taken during the optically thin phase. $\mathrm{H} \beta$ is present, although its strength slowly decreases at $>250 \mathrm{~d}$.
The Fe II lines around $5000 \AA$ are weak and hard to distinguish. The [OI] $\lambda \lambda 6300,6364$ doublet has two distinguishable components (separated by $\sim 62 \AA$ ) and appears after 5 months, becoming prominent. At 5 months, we see the presence of He I $\lambda 7065$, [Fe II] $\lambda 7155$, [Ca II] $\lambda \lambda 7291,7323$ and [Ni II] $\lambda 7378$, which become prominent at later epochs. Despite being an LL SN II, SN 2016aqf displays blended [Ca II] $\lambda \lambda 7291,7323$ lines. The presence of OI $\lambda 7774$ is more prominent at these later epochs. The Ca II NIR lines are easy to distinguish, given the narrow profiles.

The [O I] $\lambda \lambda 6300,6364$ and [Ca II] $\lambda \lambda 7291,7323$ lines show some very minor redshift $\left(\sim 5 \AA\right.$, or $\sim 230 \mathrm{~km} \mathrm{~s}^{-1}$ and $\left.\sim 200 \mathrm{~km} \mathrm{~s}^{-1}\right)$, while the $\mathrm{He}$ I $\lambda 7065$, [Fe II] $\lambda 7155$, and $[\mathrm{Ni} \mathrm{II}] \lambda 7378$ lines are more redshifted $\left(\sim 15 \AA\right.$, or $\sim 630 \mathrm{~km} \mathrm{~s}^{-1}, \sim 630 \mathrm{~km} \mathrm{~s}^{-1}$, and $\sim 610 \mathrm{~km} \mathrm{~s}^{-1}$ ) throughout most of the nebular phase. We also noticed that the [Ni II] $\lambda 7378$ line shows almost no redshift $\left(\sim 2 \AA\right.$, or $\left.\sim 80 \mathrm{~km} \mathrm{~s}^{-1}\right)$ at $\sim+150 \mathrm{~d}$ before rapidly increasing to $\sim 10 \AA\left(\sim 400 \mathrm{~km} \mathrm{~s}^{-1}\right)$ at $\sim+165 \mathrm{~d}$ and $\sim 20 \AA\left(\sim 800 \mathrm{~km} \mathrm{~s}^{-1}\right)$ at $\sim+270 \mathrm{~d}$. In addition, the [O I] $\lambda \lambda 6300,6364$ lines show a minor blueshift $\left(\sim 5 \AA, \sim 230 \mathrm{~km} \mathrm{~s}^{-1}\right)$ at $\sim+280 \mathrm{~d}$ and then get blueshifted again in about 1 month. These shifts could be caused by asymmetries caused by clumps in different layers of the expanding envelope. It is worth mentioning that the $[\mathrm{Fe} \mathrm{II}] \lambda 7172$ and $[\mathrm{Ni} \mathrm{II}] \lambda 7412$ lines can contribute to the shifts in the [Fe II] $\lambda 7155$ and [Ni II] $\lambda 7378$ lines, respectively. However, due to the resolution of the spectra, we are unable to discern their contribution. Table 5 contains a list of lines and FWHM measurements of SN 2016aqf.

When we compare SN2016aqf to other SNe at $>+300 \mathrm{~d}$ (see Fig. 8), some of them do not show He I $\lambda 7065$ (e.g. SN 2005cs and SN 2012ec). For SN 2009N, which does show this line, it has a similar strength to [Fe II] $\lambda 7155$, which does not occur for other 


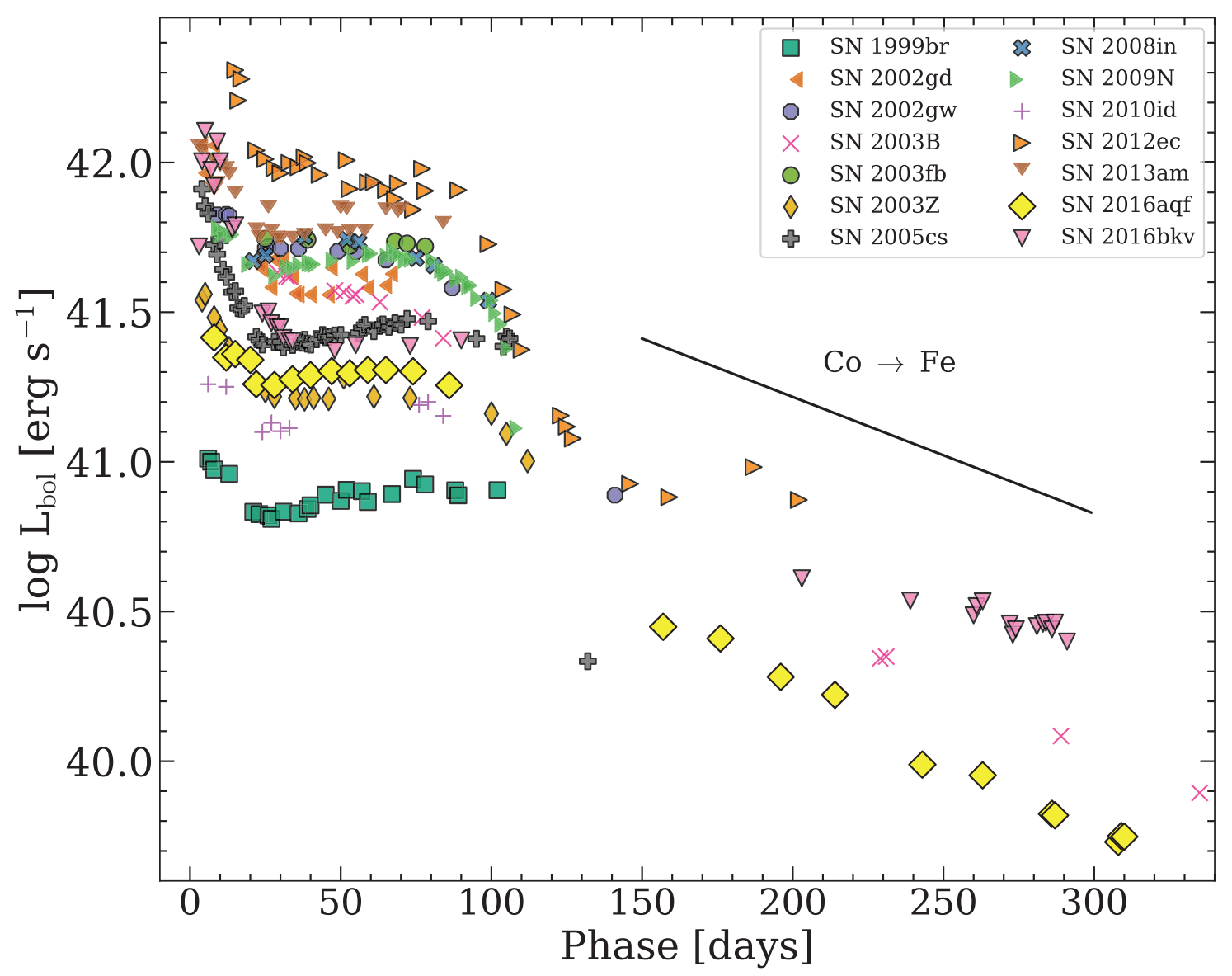

Figure 4. Bolometric light curve of SN 2016aqf compared to our LL SNe II sample. The light curves were obtained by using bolometric corrections (see Section 3.4 for details). All data are corrected for MW and host galaxy extinction (except for those with values reported as upper limits, see Table 3). The ${ }^{56}$ Co $\rightarrow{ }^{56} \mathrm{Fe}$ decay line is shown for comparison. Uncertainties are not shown for visualization purposes.

SNe. The ratio between the [O I] $\lambda \lambda 6300,6364$ lines is similar for all SNe, except for SN 2005 cs where these lines have a similar flux. It can also be seen that [Ni II] $\lambda 7378$ is easy to distinguish in some SNe (e.g. SN 2012ec, SN 2009N, and SN 2016aqf). In the case of SN 2003B and SN 2005cs, this line is present, but it gets blended with the [Ca II] $\lambda \lambda 7291,7323$ doublet. SN 2012ec is a special case as it is the only SN that shows a higher peak in [Ni II] $\lambda 7378$ than in the [Ca II] $\lambda \lambda 7291,7323$ doublet.

\subsection{Expansion velocity evolution}

The ejecta expansion velocities were measured from the position of the absorption minima for $\mathrm{H} \beta, \mathrm{Fe}$ II $\lambda 4924$, Fe II $\lambda 5018$, Fe II $\lambda 5169$, Na I D (middle of the doublet), Ba II $\lambda 6142$, Sc II $\lambda 6247$, and $\mathrm{H} \alpha$. For $\mathrm{H} \alpha$, we also estimated the expansion velocity from the FWHM (corrected for the instrumental resolution) of the emission by using $v=c \times \mathrm{FWHM} / \lambda_{\text {rest }}$, where $c$ is the speed of light. We include uncertainties in the measurement of the absorption minima, from the host galaxy recession velocity $\left(3 \mathrm{~km} \mathrm{~s}^{-1}\right.$, as reported in HyperLEDA ${ }^{7}$; Makarov et al. 2014), the maximum rotation velocity of the galaxy (44.2 $\mathrm{km} \mathrm{s}^{-1}$, as reported in HyperLEDA) and from the instrumental resolution, all added in quadrature. The major contribution to the uncertainty comes from the instrumental resolution.

\footnotetext{
${ }^{7}$ http://leda.univ-lyon1.fr
}

The expansion velocity curves are shown in Fig. 9. The velocities of $\mathrm{H} \alpha$ and $\mathrm{H} \beta$ are relatively high $\left(\gtrsim 8000 \mathrm{~km} \mathrm{~s}^{-1}\right)$ at very early epochs $(t \lesssim 10 \mathrm{~d})$ and drop to $\sim 5000$ and $4000 \mathrm{~km} \mathrm{~s}^{-1}$ at $\sim 50 \mathrm{~d}$, respectively, decreasing at a slower rate afterwards. The $\mathrm{H} \alpha$ velocity estimated from the FWHM is close to that estimated from the absorption minima as shown by Gutiérrez et al. (2017). The velocities of other lines decrease less dramatically, from $\sim 5000 \mathrm{~km} \mathrm{~s}^{-1}$ at early epochs $(t \sim 10 \mathrm{~d})$, for the Fe II lines, dropping down to $\sim 3000 \mathrm{~km}$ $\mathrm{s}^{-1}$ at $\sim 50 \mathrm{~d}$, and then constant thereafter.

In general, the expansion velocity curves of SN 2016aqf fall within the bulk of our sample and follow the general trend, although some of the velocities seem to decrease faster during the first $50 \mathrm{~d}$ after explosion.

\section{PHYSICAL PARAMETERS}

\subsection{Nickel mass}

The $\mathrm{M}_{\mathrm{Ni}}$ is one of the main physical parameters that characterize $\mathrm{CCSNe}$ as it is formed very close to the core (within a few thousand kilometres; e.g. Kasen \& Woosley 2009). We estimated the nickel mass of SN 2016aqf by using different methods. These come from: (i) Arnett (1996), (ii) Hamuy (2003), (iii) Maguire et al. (2012), and (iv) Jerkstrand et al. (2012). For more information regarding the different relations used for the estimation of the nickel mass, see Appendix A. 


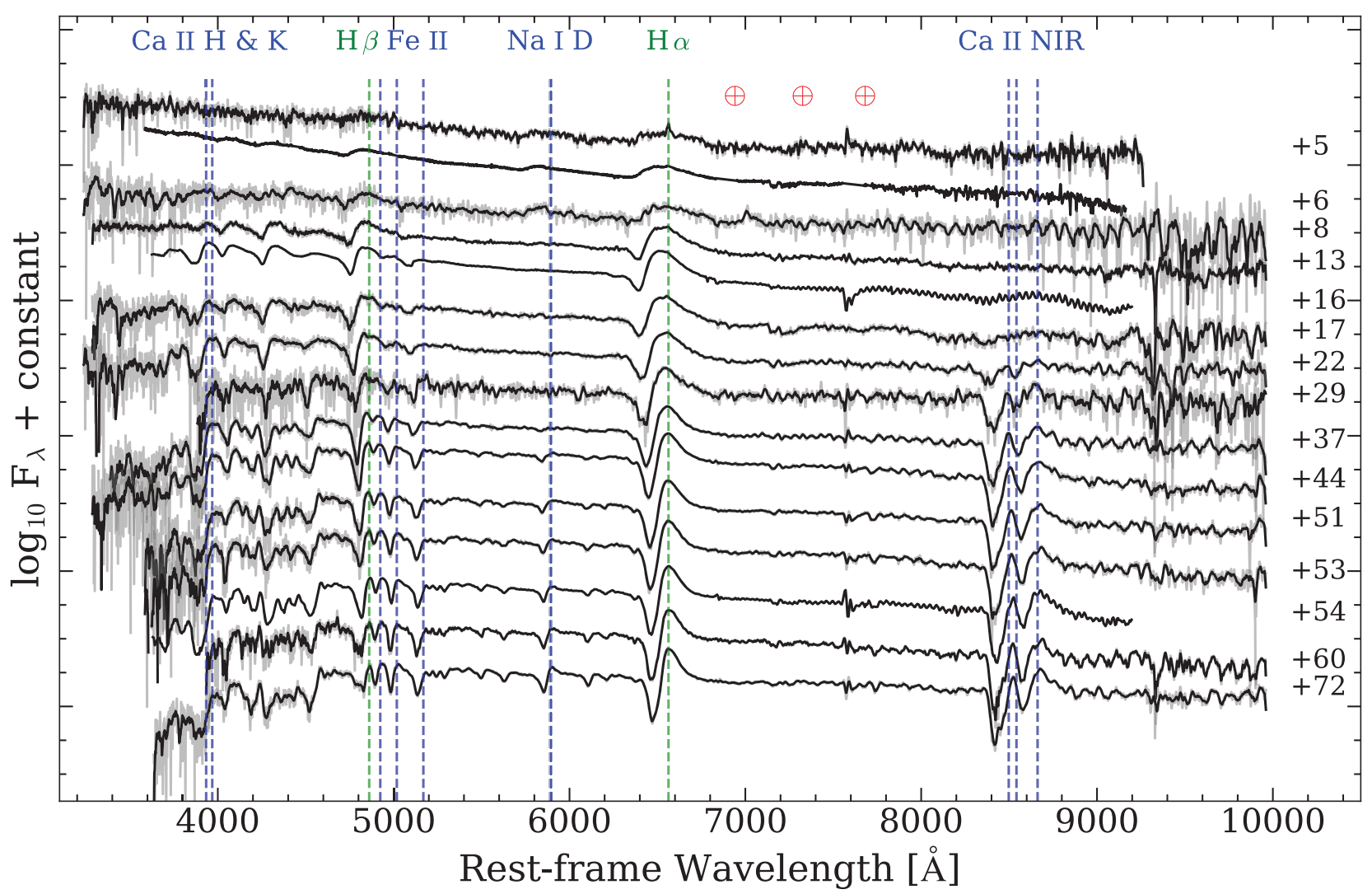

Figure 5. SN 2016aqf photospheric phase spectra. Ca II H\&K, H $\beta$, Fe II $\lambda \lambda \lambda 4924,5018,5169$, Na I D, H $\alpha$, and Ca II NIR lines are marked. Green vertical lines denote single lines, while blue denotes doublets or triplets. Telluric lines are shown by red circles with crosses. In some cases, the binned spectra (black line) are over-plotted on the original spectra (grey) for visualization. Spectra corrected for MW extinction.
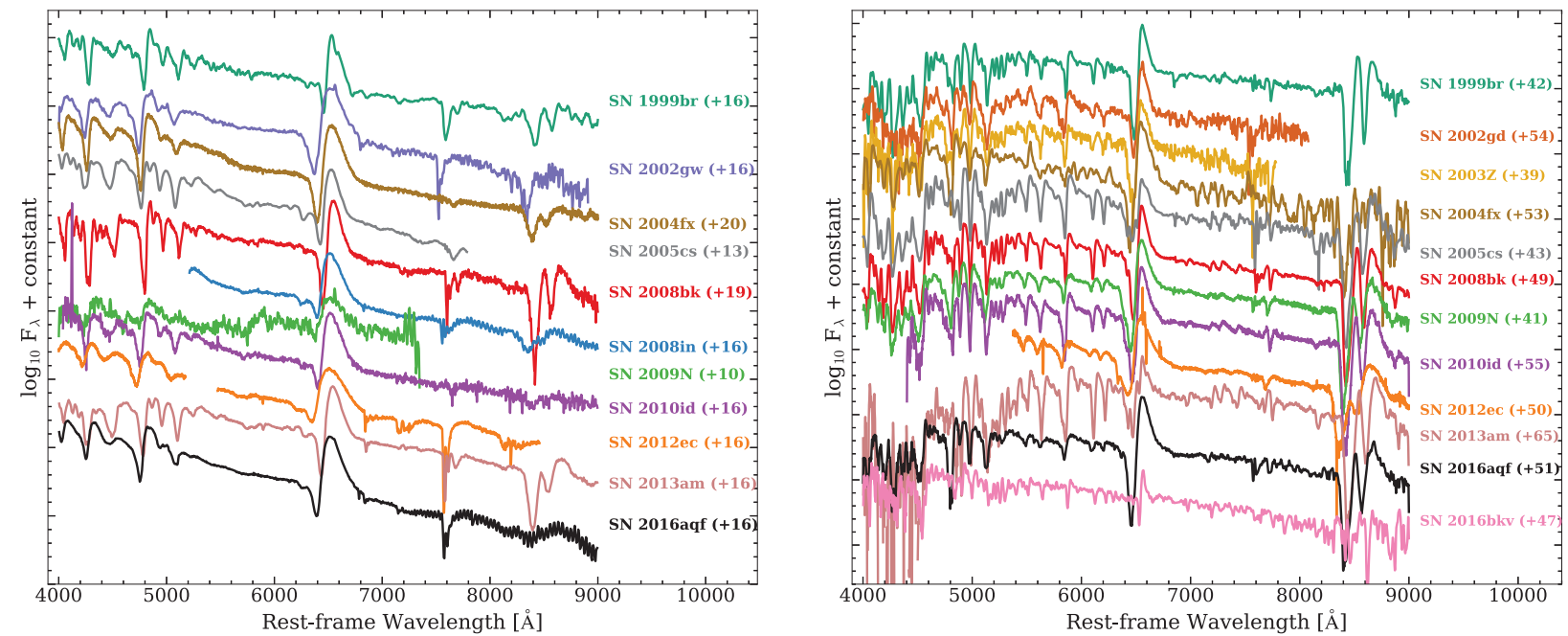

Figure 6. SN 2016aqf spectrum around $+15 \mathrm{~d}($ left $)$ and $+50 \mathrm{~d}$ (right) compared with the LL SNe II sample at similar epochs. Spectra corrected for MW and host galaxy extinction (except for those with values reported as upper limits, see Table 3 ).

For (i), (ii), and (iv), we used the bolometric luminosity of the exponential decay tail at $+200 \mathrm{~d}$, calculated in Section 3.4 by interpolating with Gaussian Process (Rasmussen \& Williams 2006), using the PYTHON package GEORGE ${ }^{8}$ (Ambikasaran et al.

${ }^{8} \mathrm{https}: / /$ github.com/dfm/george
2015) and including the distance of the $\mathrm{SN}$ for (ii). In the case of (iii), we measured the FWHM of $H_{\alpha}$ at $+351 \mathrm{~d}$, correcting it for the FWHM of the instrument. The $\mathrm{M}_{\mathrm{Ni}}$ values obtained with the different methods were $\mathrm{M}_{\mathrm{Ni}}=0.008_{-0.002}^{+0.002}, 0.011_{-0.003}^{+0.003}, 0.014_{-0.007}^{+0.009}$ and $0.007_{-0.001}^{+0.001} \mathrm{M}_{\odot}$, respectively. Using the different methods, we estimated a weighted mean and a weighted standard error of the mean of $\mathrm{M}_{\mathrm{Ni}}=0.008 \pm 0.002 \mathrm{M}_{\odot}$. 
Table 4. pEW for several lines during the optically thick phase and $\mathrm{H}_{\alpha}$ FWHM. These values are not corrected for instrument resolution. Phase with respect to the explosion epoch.

\begin{tabular}{|c|c|c|c|c|c|c|c|c|c|}
\hline Phase & $\begin{array}{c}\mathrm{pEW}\left(\mathrm{H}_{\beta}\right) \\
(\AA)\end{array}$ & $\begin{array}{c}\mathrm{pEW}(\mathrm{Fe} \text { II } 4924) \\
(\AA)\end{array}$ & $\begin{array}{c}\mathrm{pEW}(\mathrm{Fe} \text { II 5018) } \\
(\AA)\end{array}$ & $\begin{array}{c}\mathrm{pEW}(\mathrm{Fe} \text { II } 5169) \\
(\AA)\end{array}$ & $\begin{array}{c}\mathrm{pEW}(\mathrm{Na} \text { I D) } \\
(\AA)\end{array}$ & $\begin{array}{c}\mathrm{pEW}(\mathrm{Ba} \mathrm{II} \mathrm{6142)} \\
(\AA \AA)\end{array}$ & $\begin{array}{c}\mathrm{pEW}(\mathrm{Sc} \text { II 6247) } \\
(\AA)\end{array}$ & $\begin{array}{c}\mathrm{pEW}\left(\mathrm{H}_{\alpha}\right) \\
(\AA)\end{array}$ & $\begin{array}{c}\mathrm{FWHM}\left(\mathrm{H}_{\alpha}\right) \\
(\AA)\end{array}$ \\
\hline 13 & $31.7 \pm 3.1$ & - & - & - & - & - & - & $19.0 \pm 0.9$ & $189.7 \pm 2.5$ \\
\hline 16 & $34.0 \pm 2.0$ & - & $1.3 \pm 0.1$ & $12.7 \pm 0.4$ & - & - & - & $29.2 \pm 3.2$ & $170.0 \pm 2.6$ \\
\hline 22 & $51.0 \pm 2.6$ & - & $16.3 \pm 0.6$ & $19.3 \pm 1.5$ & - & - & - & $46.0 \pm 2.0$ & $156.0 \pm 1.7$ \\
\hline 29 & $32.3 \pm 1.5$ & - & - & - & - & - & - & $62.0 \pm 7.0$ & $140.0 \pm 5.2$ \\
\hline 37 & $37.7 \pm 1.2$ & $6.3 \pm 0.8$ & $16.2 \pm 0.8$ & $23.7 \pm 7.6$ & $6.9 \pm 1.3$ & $3.2 \pm 0.5$ & $4.1 \pm 1.0$ & $62.3 \pm 4.5$ & $113.0 \pm 6.0$ \\
\hline 44 & $43.3 \pm 1.5$ & $8.2 \pm 0.4$ & $19.3 \pm 1.5$ & $31.3 \pm 2.3$ & $9.4 \pm 0.9$ & $5.1 \pm 0.4$ & $3.9 \pm 0.7$ & $65.7 \pm 3.2$ & $100.3 \pm 6.1$ \\
\hline 54 & $55.0 \pm 3.6$ & $11.7 \pm 0.5$ & $20.3 \pm 0.6$ & $33.7 \pm 2.1$ & $24.0 \pm 1.7$ & $8.9 \pm 1.6$ & $5.0 \pm 0.4$ & $68.7 \pm 1.5$ & $94.0 \pm 3.0$ \\
\hline 60 & $45.3 \pm 2.9$ & $14.3 \pm 0.5$ & $23.0 \pm 1.0$ & $37.0 \pm 3.0$ & $24.0 \pm 2.6$ & $11.0 \pm 1.1$ & $7.1 \pm 0.3$ & $66.0 \pm 2.6$ & $88.3 \pm 2.5$ \\
\hline 71 & $37.0 \pm 1.7$ & $17.7 \pm 0.6$ & $25.0 \pm 1.0$ & $40.3 \pm 1.5$ & $30.7 \pm 2.1$ & $15.3 \pm 1.2$ & $7.2 \pm 0.5$ & $62.0 \pm 2.6$ & $82.7 \pm 5.0$ \\
\hline
\end{tabular}

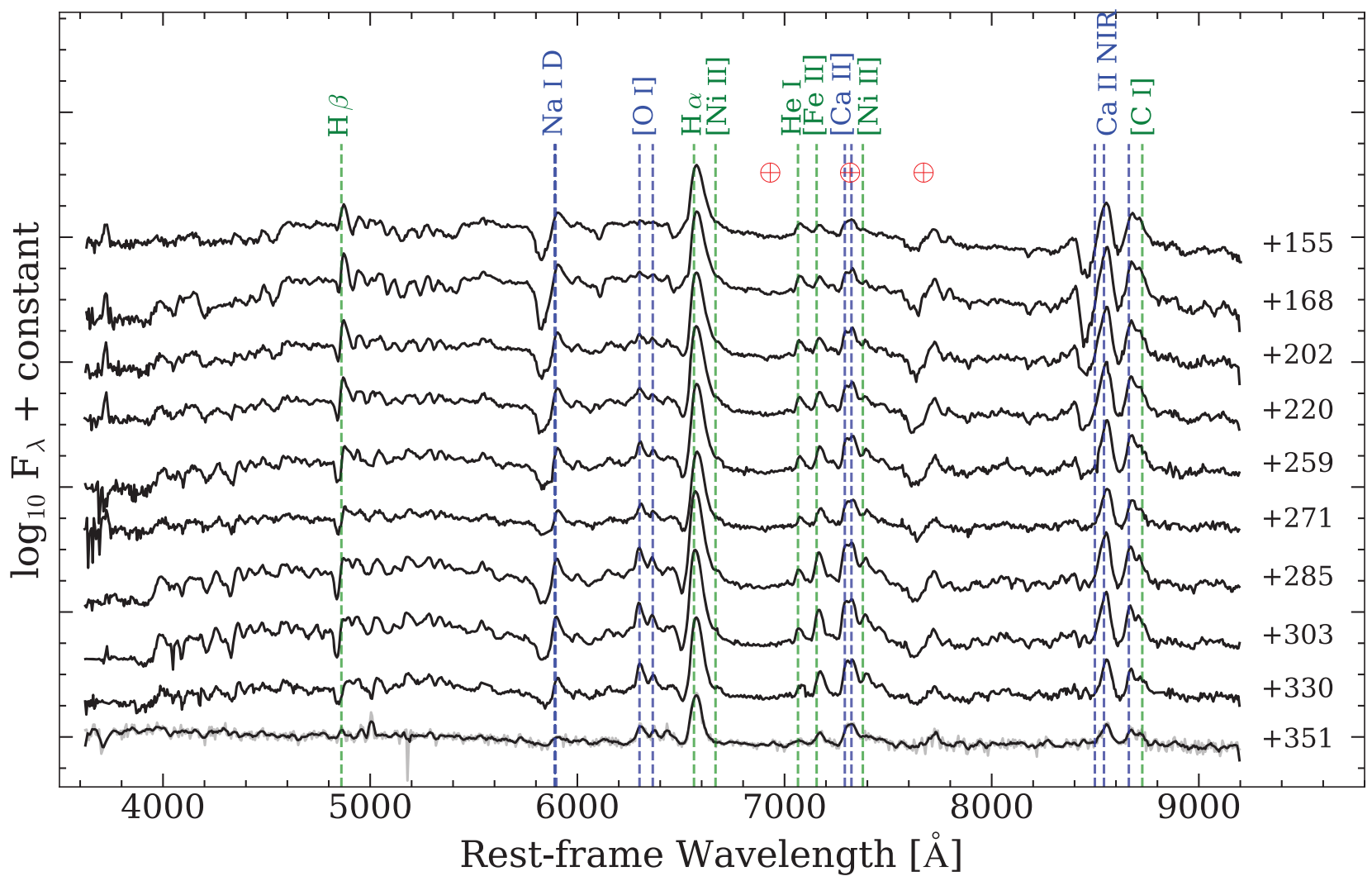

Figure 7. SN 2016aqf nebular phase spectroscopy. $\mathrm{H} \alpha, \mathrm{H} \beta, \mathrm{Na}$ I D, [O I] $\lambda \lambda 6300,6364$, [Ni II] $\lambda 6667, \mathrm{He}$ I $\lambda 7065$, [Fe II] $\lambda 7155$, [Ca II] $\lambda \lambda 7291,7323$, [Ni II] $\lambda 7378$, and Ca II NIR lines are shown for guidance. The rest of the description is the same as in Fig. 5.

Table 5. FWHM for lines during the optically thin phase. Values are corrected for the instrument resolution.

\begin{tabular}{lccccc}
\hline $\begin{array}{l}\text { Phase } \\
(\mathrm{d})\end{array}$ & $\begin{array}{c}\text { FWHM([O I] 6300) } \\
(\AA)\end{array}$ & $\begin{array}{c}\text { FWHM([O I] 6364) } \\
(\AA)\end{array}$ & $\begin{array}{c}\text { FWHM }\left(\mathrm{H}_{\alpha}\right) \\
(\AA)\end{array}$ & $\begin{array}{c}\text { FWHM(He I 7065) } \\
(\AA)\end{array}$ & $\begin{array}{c}\text { FWHM([Fe II] 7155) } \\
(\AA)\end{array}$ \\
\hline 155 & - & - & $43.8 \pm 0.6$ & $47.8 \pm 3.1$ & $41.2 \pm 2.1$ \\
168 & $29.5 \pm 2.1$ & $18.7 \pm 2.1$ & $40.0 \pm 0.6$ & $36.3 \pm 2.6$ & $36.3 \pm 2.0$ \\
202 & $33.6 \pm 1.5$ & $20.8 \pm 2.1$ & $40.5 \pm 0.6$ & $30.3 \pm 1.0$ & $34.3 \pm 1.2$ \\
220 & $38.2 \pm 6.7$ & $23.6 \pm 1.2$ & $38.2 \pm 0.6$ & $32.7 \pm 1.0$ & $34.7 \pm 1.5$ \\
259 & $24.9 \pm 2.5$ & $17.8 \pm 1.2$ & $37.8 \pm 0.6$ & $24.4 \pm 1.5$ & $34.3 \pm 1.5$ \\
271 & $24.4 \pm 2.5$ & $20.8 \pm 2.1$ & $39.4 \pm 0.6$ & $23.0 \pm 1.5$ & $34.7 \pm 1.5$ \\
285 & $27.5 \pm 2.1$ & $20.8 \pm 3.8$ & $35.9 \pm 0.6$ & $28.7 \pm 1.5$ & $32.4 \pm 1.2$ \\
303 & $28.2 \pm 2.5$ & $22.2 \pm 4.0$ & $35.4 \pm 0.6$ & $27.0 \pm 0.6$ & $29.5 \pm 1.5$ \\
330 & $28.2 \pm 1.5$ & $24.9 \pm 2.1$ & $35.9 \pm 0.6$ & $28.2 \pm 2.1$ & $34.7 \pm 1.2$ \\
351 & $39.7 \pm 3.6$ & $19.8 \pm 4.4$ & $37.8 \pm 0.6$ & $70.9 \pm 9.0$ & $43.4 \pm 4.0$ \\
\hline
\end{tabular}




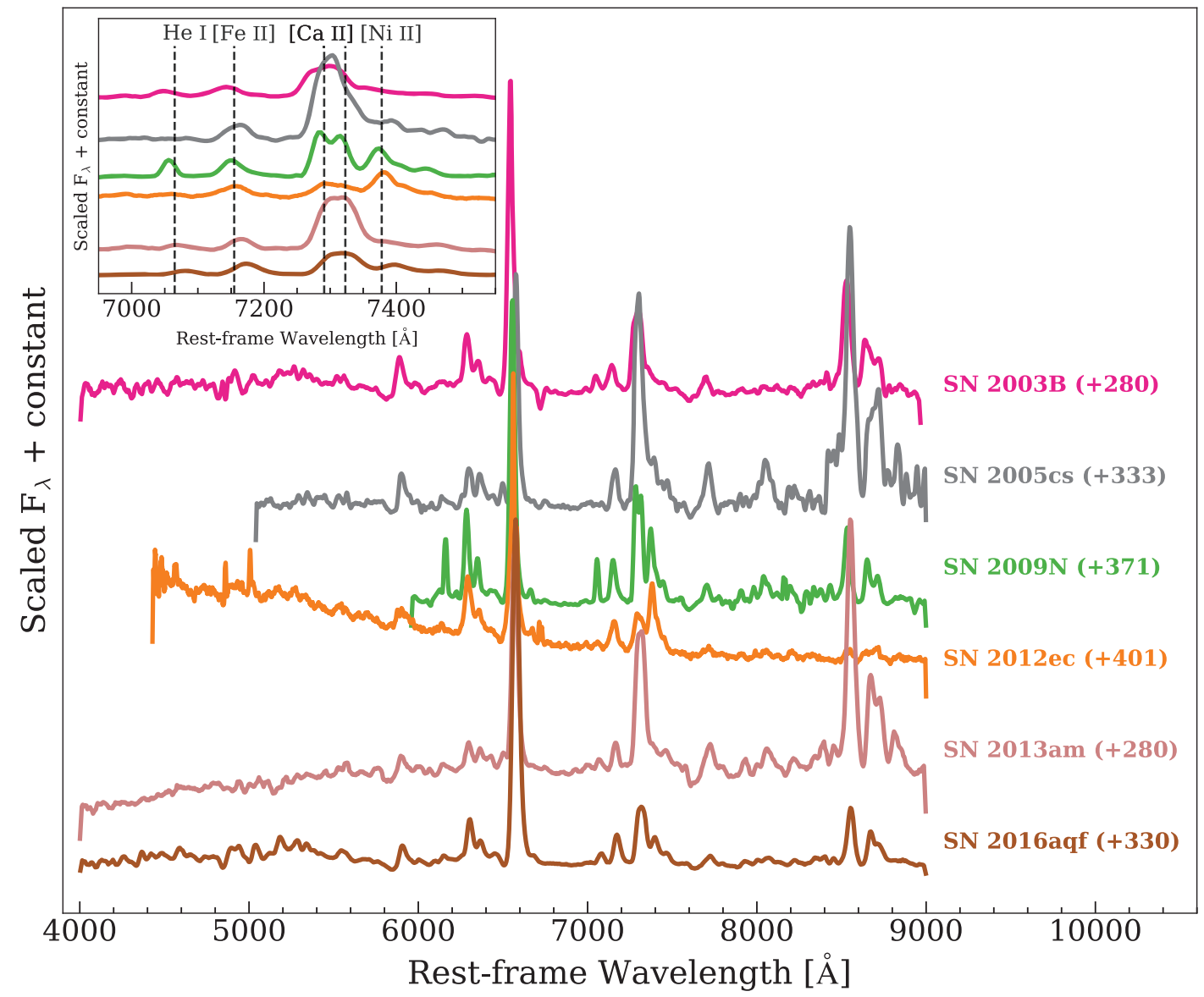

Figure 8. SN 2016aqf spectrum around $+330 \mathrm{~d}$ compared with the LL SN II sample at similar epochs. The spectra were normalized by their peak H $\alpha$ flux. Embedded figure: zoom-in around $\sim 7250 \AA$ A. The rest-frame position of the He I $\lambda 7065$, [Fe II] $\lambda 7155$, [Ca II] $\lambda \lambda 7291,7323$ and [Ni II] $\lambda 7378$ lines is shown. Spectra corrected for MW host galaxy extinction (except for those with values reported as upper limits, see Table 3).

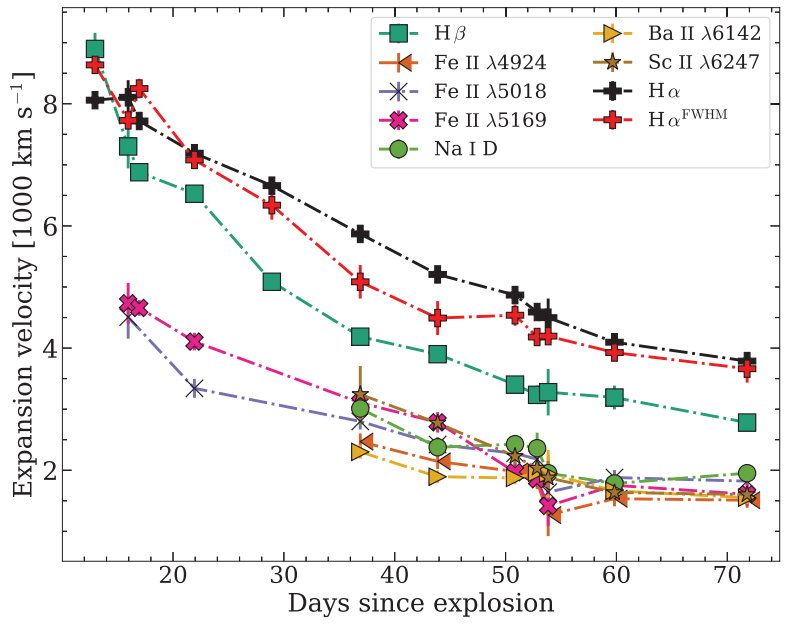

Figure 9. SN 2016aqf expansion velocities for $\mathrm{H} \beta$, Fe II $\lambda 4924$, Fe II $\lambda 5018$, Fe II $\lambda 5169$, Na I D (middle of the doublet), Ba II $\lambda 6142$, Sc II $\lambda 6247$, and $\mathrm{H} \alpha$. For $\mathrm{H} \alpha$, we also estimated the expansion velocity from the FWHM; see text for details.

\subsection{Explosion energy, ejected mass, and progenitor radius}

Popov (1993) derived analytical relations for the estimation of the explosion energy $\left(E_{\text {exp }}\right)$, ejected envelope mass $\left(M_{\text {env }}\right)$, and the progenitor radius prior to outburst $\left(\mathrm{R}_{\text {prog }}\right)$ for $\mathrm{SNe}$ II-P (following a similar analysis by Litvinova \& Nadezhin 1985). These parameters are related to different light-curve properties and also $\mathrm{M}_{\mathrm{Ni}}$; therefore, they are essential for the characterization of SNe II and CCSNe in general. The relations found by Popov (1993) are

$\log _{10}\left(\mathrm{E}_{\mathrm{exp}}\right)=4.0 \log _{10} t_{\mathrm{p}}+0.4 M_{V}+5.0 \log _{10}\left(v_{\mathrm{ph}}\right)-4.311$,

$\log _{10}\left(\mathrm{M}_{\mathrm{env}}\right)=4.0 \log _{10} t_{\mathrm{p}}+0.4 M_{V}+3.0 \log _{10}\left(v_{\mathrm{ph}}\right)-2.089$,

and

$\log _{10}\left(\mathrm{R}_{\text {prog }}\right)=-2.0 \log _{10} t_{\mathrm{p}}-0.8 M_{V}-4.0 \log _{10}\left(v_{\mathrm{ph}}\right)-4.278$,

where $M_{V}$ is the $V$-band absolute magnitude at the middle of the plateau, $t_{\mathrm{p}}$ is the duration of the plateau in days (as in Hamuy 2003), and $v_{\mathrm{ph}}$ is the expansion velocity of the photosphere at $t_{\mathrm{p}} / 2$ (usually measured from the Fe II $\lambda 5169$ line, as it has shown to be a good tracer of the photosphere) in $10^{3} \mathrm{~km} \mathrm{~s}^{-1}$. $\mathrm{E}_{\exp }$ is expressed in $10^{51} \mathrm{erg}$, and $\mathrm{M}_{\mathrm{env}}$ and $\mathrm{R}_{\text {prog }}$ in solar units. We measured $\mathrm{M}_{V}=-14.63 \pm 0.27$ mag for which we used Gaussian processes to interpolate the light curve. By using the relativistic Doppler shift, we obtained $v_{\mathrm{ph}}=$ $2068 \pm 167 \mathrm{~km} \mathrm{~s}^{-1}$ from the Fe II $\lambda 5169$ absorption line minima. Finally, we use $t_{\mathrm{p}}=97.9 \pm 7.2 \mathrm{~d}$, for which we assumed the same value of SN 2003fb, adding its uncertainty (see Anderson et al. 2014) in quadrature, as these $\mathrm{SNe}$ have relatively similar evolution around 
the transition ( $t \gtrsim+50 \mathrm{~d})$ in the $V$ band (see Appendix B). With these values for SN 2016aqf, we obtained $\mathrm{E}_{\text {exp }}=0.24 \pm 0.13 \times 10^{51}$ erg, $M_{\text {env }}=9.31 \pm 4.26 \mathrm{M}_{\odot}$, and $\mathrm{R}_{\text {prog }}=152 \pm 94 \mathrm{R}_{\odot}$. The large uncertainties come mainly from the velocity, specifically from the instrumental resolution, and from the distance uncertainty used in calculating the absolute magnitude. We compared these results with similar relations found in the literature (e.g. Kasen \& Woosley 2009; Shussman et al. 2016; Sukhbold et al. 2016; Goldberg, Bildsten \& Paxton 2019; Kozyreva, Nakar \& Waldman 2019; Kozyreva et al. 2020), obtaining similar results.

SN 2016aqf follows the $\mathrm{E}_{\mathrm{exp}}-\mathrm{M}_{\mathrm{Ni}}$ relation found in $\mathrm{SNe}$ II (e.g. Pejcha \& Prieto 2015; Müller et al. 2017), and $M_{\text {env }}$ follows the $\mathrm{M}_{\mathrm{env}}-\mathrm{E}_{\mathrm{exp}}$ relation (e.g. Pejcha \& Prieto 2015). If we assume a neutron star $\left(\sim 1.4 \mathrm{M}_{\odot}\right)$ as the compact remnant, the progenitor of SN 2016aqf should be an RSG with $\sim 10.7 \mathrm{M}_{\odot}$. This is a lower limit, as some mass loss is expected due to various processes, e.g. winds (e.g. Dessart et al. 2013b). Finally, $\mathrm{R}_{\text {prog }}$ is well within the normal values of RSG radii, although on the lower end (e.g. Pejcha \& Prieto 2015; Müller et al. 2017), but consistent with other estimations for this sub-class of SN (e.g. Chugai \& Utrobin 2000; Zampieri et al. 2003; Pastorello et al. 2009; Roy et al. 2011).

\section{DISCUSSION}

\subsection{Progenitor mass}

The progenitors of SNe II have been extensively studied through preSN images (e.g. Smartt et al. 2009; Smartt 2015) and hydrodynamical models (e.g. Bersten, Benvenuto \& Hamuy 2011; Dessart et al. 2013b; Martinez \& Bersten 2019). Although there remain some disagreements (e.g. Utrobin \& Chugai 2009; Dessart et al. 2013b, for discussions of this discrepancy), there have been recent major improvements due to better cadence observations.

The [O I] $\lambda \lambda 6300,6364$ nebular-phase lines have also been shown to be good tracers of the core mass of CCSN progenitors (e.g. Elmhamdi et al. 2003; Sahu et al. 2006; Maguire et al. 2010), as at these later epochs, we are observing deeper into the progenitor structure. Spectral modelling of the nebular phase has shown good agreement with this and can be used to estimate the progenitor mass (e.g. J12, J14, J18). In order to estimate the progenitor mass of SN 2016aqf, we used the spectral synthesis models from J14 and J18 for progenitors with three different ZAMS masses: 9, 12, and $15 \mathrm{M}_{\odot}$. The $9 \mathrm{M}_{\odot}$ model has an initial ${ }^{56} \mathrm{Ni}$ mass of $0.0062 \mathrm{M}_{\odot}$ while the other two models have an initial ${ }^{56} \mathrm{Ni}$ mass of $0.062 \mathrm{M}_{\odot}$. We compare the nebular spectra of SN 2016aqf with the models at two different epochs each (see Fig. 10 for models at +300 d). The models are scaled by $\exp \left(\left(\mathrm{t}_{\mathrm{mod}}-\mathrm{t}_{\mathrm{SN}}\right) / 111.4\right)$, where $\mathrm{t}_{\bmod }$ is the epoch of the spectrum of the models and $t_{\mathrm{SN}}$ is the epoch of the spectrum of the $\mathrm{SN}$, by the $\mathrm{SN}$ nickel mass, $\mathrm{M}_{\mathrm{Ni}}^{\mathrm{SN}} / \mathrm{M}_{\mathrm{Ni}}^{\bmod }$, and by the inverse square of the $\mathrm{SN}$ distance $\left(\mathrm{d}_{\text {mod }} / \mathrm{d}_{\mathrm{SN}}\right)^{2}$. The luminosity of some lines, like [OI] $\lambda 6300,6364$, scales relatively linearly with the $\mathrm{M}_{\mathrm{Ni}}$ (as discussed, e.g. in J14); thus, it is reasonably accurate to compare the models rescaled, with the difference in $\mathrm{M}_{\mathrm{Ni}}$, to our observed $\mathrm{SN} . \chi^{2}$ values are calculated to quantify these comparisons as well.

From Fig. 10, we see that the 12 and $15 \mathrm{M}_{\odot}$ models present similar results, reproducing several lines. They can partially reproduce the [O I] $\lambda 6300$ line, but the latter does not reproduce the [O I] $\lambda 6364$ line very well. However, these models under-predict the $[\mathrm{Fe}$ II $] \lambda 7155$ line and do not reproduce the $[\mathrm{Ni}$ II] $\lambda 7378$ line and Ca II NIR triplet. The $9 \mathrm{M}_{\odot}$ mostly over-predicts the flux of lines but does a good job reproducing the $\mathrm{He} \mathrm{I} \lambda 7065$ and [Fe II] $\lambda 7155$ lines. In terms of $\chi^{2}$ values, the $12 \mathrm{M}_{\odot}$ model is slightly better than the $15 \mathrm{M}_{\odot}$ one, while the $9 \mathrm{M}_{\odot}$ model has a poorer fit. In addition, the $12 \mathrm{M}_{\odot}$ model is relatively consistent with the mass estimate from Section 4.2, within the uncertainty. We also measured [O I]/[Ca II] flux ratios (e.g. Maguire et al. 2010) between $\sim 0.5$ and 0.7 , which are consistent with the $12 \mathrm{M}_{\odot}$ model and roughly consistent with the $15 \mathrm{M}_{\odot}$ model. Finally, we found that the models reproduce lines better at later epochs ( $\gtrsim 300 \mathrm{~d})$ than at early epochs $(<300 \mathrm{~d})$. J18 found the same pattern.

There seems to be a very weak detection of [Ni II] $\lambda 6667$ (see Fig. 7), partially blended with $\mathrm{H} \alpha$, and the $9 \mathrm{M}_{\odot}$ model predicts similar fluxes for this line and [Ni II] $\lambda 7378$, due to the high optical depths (fig. 20 of J18). Note that this model has only primordial nickel in the hydrogen zone, no synthesized ${ }^{58} \mathrm{Ni}$, and a different setup compared to the other two (e.g. no mixing applied, J18). As the model prediction for [Ni II] $\lambda 7378$ is too weak, one can argue the detection of synthesized nickel. The $9 \mathrm{M}_{\odot}$ model overpredicts the $[\mathrm{OI}] \lambda 6300,6364$ lines, including most other lines. As mentioned above, J18 had similar results at these early epochs; however, this model showed better agreement at later epochs (e.g. $>350 \mathrm{~d}$ for SN 2005cs). We did not find better agreement at later epochs.

In order to expand our analysis, we also compared SN 2016aqf with the progenitor models from Lisakov et al. (2017), specifically, the YN models of $12 \mathrm{M}_{\odot}$ (a set of piston-driven explosion with ${ }^{56} \mathrm{Ni}$ mixing), as their $\mathrm{M}_{\mathrm{Ni}}\left(0.01 \mathrm{M}_{\odot}\right)$ agree perfectly with our estimation, apart from agreeing with other physical parameters (e.g. $\mathrm{E}_{\text {exp }}=2.5 \times 10^{50} \mathrm{erg}, \mathrm{M}_{\mathrm{env}}=9.45 \mathrm{M}_{\odot}$ ) as well. This comparison, which was done in the same way as with the other models above, is shown in Fig. 10 for the YN2 model as well. As can be seen, the model predicts some of the $\mathrm{Ca}$ and the $[\mathrm{OI}] \lambda 6300,6364$ lines relatively well. None the less, most of the other lines are over-predicted. Other models from Lisakov et al. (2017) did not show better agreement. However, the fact that both $12 \mathrm{M}_{\odot}$ models (from J14 and Lisakov et al. 2017) partially agree with the [OI] $\lambda 6300,6364$ lines (the main tracers of the ZAMS mass) strengthens the conclusion that the progenitor is probably a $\sim 12 \mathrm{M}_{\odot}$ RSG star.

We would like to emphasize that neither the $9 \mathrm{M}_{\odot}$ model from J18 nor the YN $12 \mathrm{M}_{\odot}$ models from Lisakov et al. (2017) have macroscopic mixing. The consistent overproduction of narrow core lines in both models (see Fig. 10) suggests that mixing is necessary, which the models from J14 have.

In conclusion, this shows that the current models have problems predicting the observed diversity of LL SNe II, probably due to the incomplete physics behind these explosions (e.g. assumptions of mixing, ${ }^{56} \mathrm{Ni}$ mass, rotation). In other words, there is a need of more models with different parameters that can help to understand the observed behaviour of these SNe. As such, we cannot exclude a $9 \mathrm{M}_{\odot}$ or a $15 \mathrm{M}_{\odot}$ progenitor. Thus, we conclude that the progenitor of SN 2016aqf had a ZAMS mass of $12 \pm 3 \mathrm{M}_{\odot}$. A more detailed modelling of the progenitor is needed to improve these constrains, although this is beyond the scope of this work.

\subsection{He I $\lambda 7065$}

The HeI $\lambda 7065$ nebular line has been studied with theoretical modelling (e.g. Dessart et al. 2013a, J18), giving a diagnostic of the He shell. These models predict the appearance of this line in $\mathrm{SNe}$ II with low mass progenitors as more massive stars have more extended oxygen shell, shielding the He shell from gamma-ray deposition. However, some LL SNe II do not show this line in their spectra 


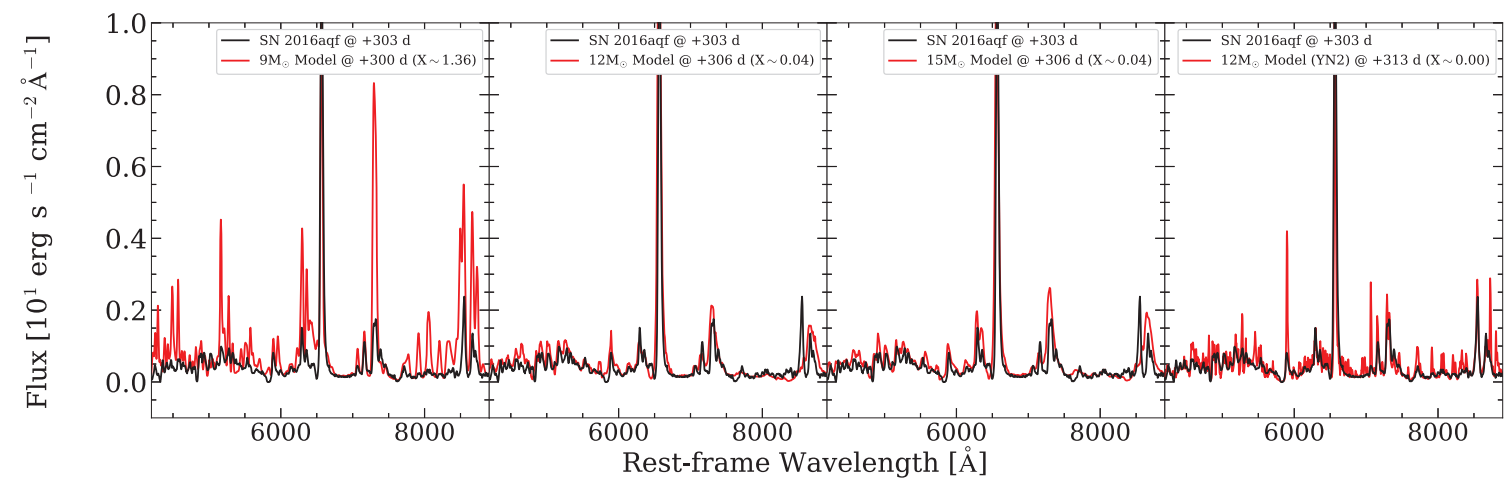

Figure 10. First three panels (from left to right): Spectral synthesis models of SNe II from J14 and J18. Three spectral synthesis models at $\sim+300 \mathrm{~d}$ from different progenitor masses: 9 (left-hand panel), 12 (centre), and $15 \mathrm{M}_{\odot}$ (right). $X$ is the scaling factor (see Section 5.1). The 12 and $15 \mathrm{M}_{\odot}$ models fit the spectrum better than the $9 \mathrm{M}_{\odot}$ model, including the [O I] $\lambda \lambda 6300,6364$ lines. Last panel: YN2 model of $12 \mathrm{M}_{\odot}$ from Lisakov et al. (2017). There is a relatively good agreement with some of the $\mathrm{Ca}$ and the [O I] $\lambda 6300,6364$ lines; however, most other lines are over-predicted.

(e.g. SN 2005cs; see Fig. 8). SN2016aqf shows the clear presence of He I $\lambda 7065$ throughout the entire nebular coverage. We also see the presence of $[\mathrm{C} I] \lambda 8727$, although it gets partially blended with the Ca II NIR triplet. We expect to see this carbon line as a result of the He shell burning, so the presence of both lines (He I $\lambda 7065$ and $[\mathrm{CI}] \lambda 8727$ ) is consistent with the theoretical prediction. Thus, we believe that SN 2016aqf is a good case study to provide further understanding of the He shell zone through theoretical models. Furthermore, following the discussion from J18, we conclude that this is an Fe core SN and not an electron-capture SN (ECSN), as the latter lack lines produced in the He layer.

\section{$5.3 \mathrm{Ni} / \mathrm{Fe}$ abundance ratio}

As discussed above, the nebular spectra of SNe II contain a lot of information regarding the progenitors as we are looking deeper into its structure. J15a discussed the importance of the ratio between the [Ni II] $\lambda 7378$ and [Fe II] $\lambda 7155$ lines as an indicator of the $N i / F e$ abundance ratio. These elements are synthesized very close to the progenitor core and, for this reason, their abundances get affected by the inner structure of the progenitor and the explosion dynamics. More specifically, iron-group yields are directly affected mainly by three properties: temperature, density, and neutron excess of the fuel (for a more detailed account, see J15b). For this reason, studying iron-group abundances is key to understanding SNe II.

SN 2016aqf is the only SN II to date with a relatively extensive coverage of the evolution of [Ni II] $\lambda 7378$ (most other SNe with the presence of this line have only at most $\sim 2$ epochs showing it). In Fig. 11, we show the evolution in time of the flux of [Ni II] $\lambda 7378$ and $[\mathrm{Fe} \mathrm{II}] \lambda 7155$ and their luminosity ratio. We estimated the fluxes by fitting Gaussians to the profiles. Uncertainties were estimated by repeating the measurements and assuming different continuum levels, but we do not include the uncertainty coming from the instrumental resolution in any of the measured fluxes throughout this work. However, this should not greatly affect the measurements as the spectral lines are in general much wider than the instrumental resolution (e.g. [Fe II] $\lambda 7155$ has an average FWHM of $\sim 35 \AA$ ).

We notice that the evolution of the luminosity ratio reaches a quasiconstant value after $\sim 170 \mathrm{~d}$ since the explosion. This suggests that at relatively late nebular phase, the $\mathrm{Ni} / \mathrm{Fe}$ abundance ratio is constant as the temperature should not vary much (see J15a), although clumps in the ejecta might cause deviations from the measured values. After removing the value at $\sim+155 \mathrm{~d}$ (as the $\mathrm{SN}$ might still be in the transition to the optically thin phase), we report an $\mathrm{Ni} / \mathrm{Fe}$ luminosity ratio weighted mean of 0.906 and a standard deviation of 0.062 . The standard deviation gives us a more conservative estimation of the uncertainty in the $\mathrm{Ni} / \mathrm{Fe}$ luminosity ratio than the uncertainty in the weighted mean.

We follow $\mathrm{J} 15$ a to estimate the $\mathrm{Ni}$ II/Fe II ratio and in turn the $\mathrm{Ni} / \mathrm{Fe}$ abundance ratio. From the ratio between the luminosity of the [Fe II] $\lambda 7155$ line and $\mathrm{M}_{\mathrm{Ni}}$, we then obtained a temperature constrain of $T=$ $3919_{-257}^{+215} \mathrm{~K}$. With these values, we estimated the Ni/Fe abundance ratio to be $0.081_{-0.010}^{+0.009}$, or $\sim 1.4$ times the solar ratio ( 0.056 , Lodders 2003).

However, there are several things we need to take into consideration. Contribution to the [Fe II] $\lambda 7155$ and $[\mathrm{Ni}$ II] $\lambda 7378$ lines does not come only from synthesized material but also from primordial $\mathrm{Fe}$ and $\mathrm{Ni}$ in the H-zone (J15a). The contribution can be significant ( $\sim 40$ per cent) and depends on the model and epoch. Unfortunately, the effect of primordial contamination is not easy to remove without detailed theoretical modelling. None the less, it is plausible that the [Fe II] $\lambda 7155$ and [Ni II] $\lambda 7378$ lines are greatly dominated by synthesized $\mathrm{Fe}$ and $\mathrm{Ni}$ at relatively early epochs $(\lesssim 300 \mathrm{~d})$, although we are uncertain at which epochs the effect from primordial $\mathrm{Fe}$ and $\mathrm{Ni}$ starts becoming important (J18). The line ratio can also be affected at very early epochs $(\lesssim 200 \mathrm{~d})$, as the SN can still be during the optically thick phase when opacity plays an important role.

Few other SNe have been reported to show [Ni II] $\lambda 7378$. It is possible that this line is mainly visible in LL SNe II, where the expansion velocities are lower, producing narrower deblended line profiles. However, it is also seen in non-LL SNe II, other CCSNe (e.g. SN 2006aj; Maeda et al. 2007; Mazzali et al. 2007), and Type Ia $\mathrm{SNe}$ (SNe Ia; e.g. Maeda et al. 2010). We searched for objects in our LL SN II comparison sample with spectra in which we could detect [Fe II] $\lambda 7155$ and [NiII] $\lambda 7378$ to measure the $\mathrm{Ni} / \mathrm{Fe}$ abundance ratio as for SN2016aqf. We also expanded this sample to include other LL SNe II: SN 1997D, SN 2003B, SN 2005cs, SN 2008bk, SN 2009N, and SN 2013am.

SN 1997D and SN 2008bk were not included in our initial sample as they lack good publicly available data. We also include SN 2012ec as it is a well-studied case. In the case of SN 1997D, we measured the ratio at two different epochs, but we used one (at $\sim+384 \mathrm{~d}$ ) of those, given that the other value (at $\sim+250 \mathrm{~d}$ ) had relatively large uncertainties. For SN 2009N, we took an average between the two values (at $\sim+372$ and +412 d) we were able to measure as they were relatively similar. SN 2016bkv was not included as the $\mathrm{M}_{\mathrm{N}}$ 

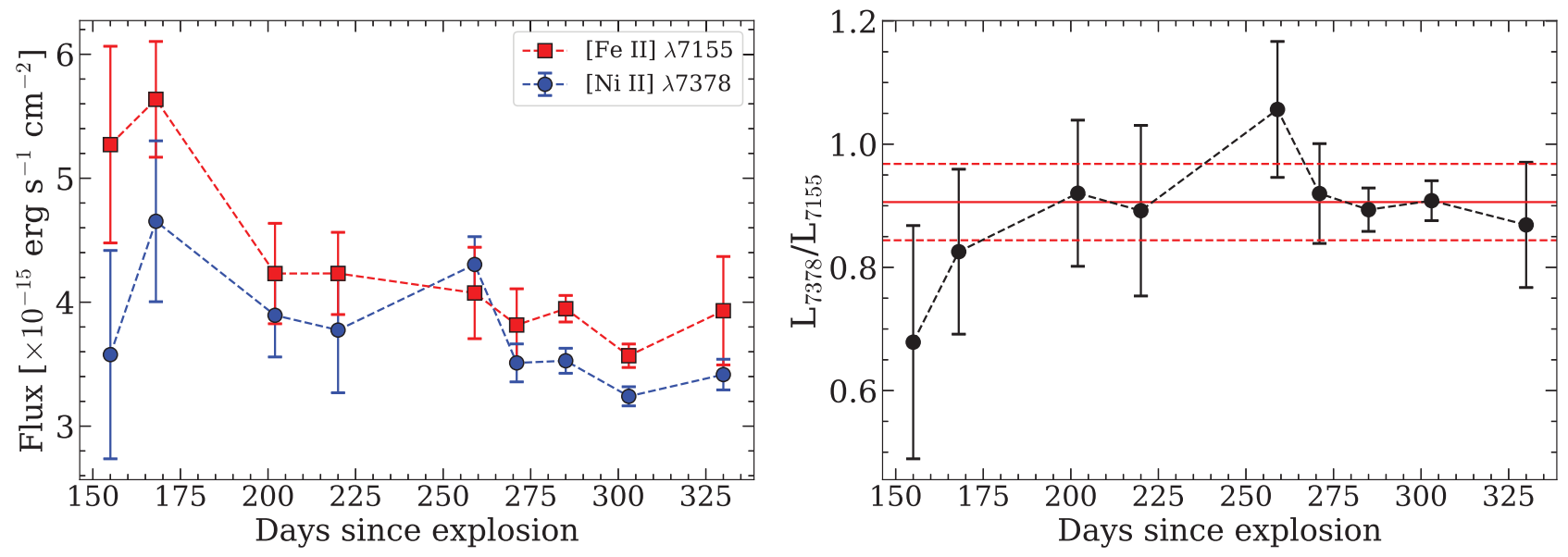

Figure 11. Left-hand panel: [Ni II] $\lambda 7378$ and [Fe II] $\lambda 7155$ lines fluxes. Right-hand panel: Luminosity ratio of these lines. The weighted average (solid red line) with a 1 standard deviation (dashed red lines) is shown for guidance. The value at $\sim+150 \mathrm{~d}$ was removed for these calculations.

values obtained in Nakaoka et al. (2018) and Hosseinzadeh et al. (2018) for this SN are not consistent with each other $\left(\sim 0.01 \mathrm{M}_{\odot}\right.$ and $0.0216 \mathrm{M}_{\odot}$, respectively), this being necessary for an accurate estimation of the $\mathrm{Ni} / \mathrm{Fe}$ abundance ratio. For the rest of the $\mathrm{SNe}$, only one value was obtained. Several other LL SNe II show the presence of [Ni II] $\lambda 7378$, but it is either blended with other lines or the $\mathrm{SNe}$ lack some of the parameters needed to estimate the $\mathrm{Ni} / \mathrm{Fe}$ abundance ratio.

To expand our analysis, we looked into other physical parameters related to the $\mathrm{Ni} / \mathrm{Fe}$ abundance ratio. For example, J15b further analyse and compare this ratio against theoretical models. Some of these models show that at lower progenitor mass, the $\mathrm{Ni} / \mathrm{Fe}$ abundance ratio should be higher. We investigate this by increasing our sample. Unfortunately, not many LL SNe II have measured progenitor masses from pre-SN images, so we added non-LL SNe II as several of these do (e.g. Smartt 2015), while they also show the presence of [Fe II] $\lambda 7155$ and [Ni II] $\lambda 7378$ in their spectra. We do not include $\mathrm{SNe}$ with estimates of the progenitor mass from other methods as they depend on more assumptions than the pre-SN images method, making these estimates less reliable. The SNe included are: SN 2007aa (Anderson et al. 2014; Gutiérrez et al. 2017), SN 2012A (Tomasella et al. 2013), and SN 2012aw (Fraser et al. 2012). All these SNe are included in Table 3. For SN 2007aa, we calculated the ejected nickel mass to be $\mathrm{M}_{\mathrm{Ni}}=0.032 \pm 0.009 \mathrm{M}_{\odot}$ (we estimated this value using the relation from Hamuy 2003 and other values from Anderson et al. 2014) and estimated the $\mathrm{Ni} / \mathrm{Fe}$ abundance ratio also as part of this work. For the other two SNe II, we took the values from $\mathrm{J} 15 \mathrm{a}$, assuming upper and lower uncertainties equal to the average of the uncertainties of the rest of the sample (not taking into account the uncertainties of SN2012ec as they are too high). The $\mathrm{Ni} / \mathrm{Fe}$ abundance ratio values for this sample are shown in Table 6

In addition, we compared the $\mathrm{Ni} / \mathrm{Fe}$ against other physical, lightcurve, and spectral parameters to investigate possible correlations. The motivation is two-fold. First, we are searching for correlations that might allow indirect methods of measuring this ratio for $\mathrm{SNe}$ with blended lines. Secondly, these correlations could shed light on the effect of different parameters in the observed value of $\mathrm{Ni} / \mathrm{Fe}$, as is expected for the progenitor mass, important for the theoretical modelling of SNe II.

We examined various parameters we thought could be somehow connected to the $\mathrm{Ni} / \mathrm{Fe}$ abundance ratio. The most relevant parameters are: nickel mass $\left(\mathrm{M}_{\mathrm{Ni}}\right)$; $\mathrm{SN} V$-band maximum absolute magnitude
Table 6. $\mathrm{Ni} / \mathrm{Fe}$ abundance ratio values used in this work

\begin{tabular}{llll}
\hline SN & $\mathrm{Ni} / \mathrm{Fe}$ & $\sigma_{-}$ & $\sigma_{+}$ \\
\hline SN1997D & 0.079 & 0.025 & 0.014 \\
SN2003B & 0.057 & 0.021 & 0.018 \\
SN2005cs & 0.084 & 0.012 & 0.012 \\
SN2007aa & 0.074 & 0.006 & 0.006 \\
SN2008bk & 0.046 & 0.042 & 0.017 \\
SN2009N & 0.101 & 0.018 & 0.017 \\
SN2012A & 0.028 & 0.022 & 0.016 \\
SN2012aw & 0.084 & 0.022 & 0.016 \\
SN2012ec & 0.2 & 0.07 & 0.07 \\
SN2013am & 0.108 & 0.017 & 0.018 \\
SN2016aqf & 0.081 & 0.010 & 0.009 \\
\hline
\end{tabular}

$\left(M_{\max }^{V}\right)$; optically thick phase duration $\left(\mathrm{OPT}_{\mathrm{d}}\right) ; \mathrm{Fe}$ II $\lambda 5169$ expansion velocity (vel(Fe II 5169)); the progenitor mass from KEPLER (K) models $\left(\mathrm{M}_{\mathrm{prog}}^{\mathrm{K}}\right.$; see Smartt 2015); progenitor mass from STARS and Geneva (SG) rotating models $\left(\mathrm{M}_{\text {prog }}^{\mathrm{SG}}\right.$; see Smartt 2015); explosion energy $\left(E_{\exp }\right) ;[\mathrm{O}$ I] $\lambda 6300$ and [O I] $\lambda 6364$ luminosities at the epoch of measured $\mathrm{Ni} / \mathrm{Fe}$ abundance ratio $\left(L_{6300}\right.$ and $\left.L_{6364}\right)$; and host galaxy gas-phase metallicity $\left((12+\log (\mathrm{O} / \mathrm{H}))_{\mathrm{N} 2}\right)$. The $\mathrm{Ni} / \mathrm{Fe}$ abundance ratio versus $\mathrm{M}_{\mathrm{Ni}}, \mathrm{M}_{\max }^{V}$ and $\mathrm{M}_{\text {prog }}$ are shown in Fig. 12.

Pearson and Spearman's rank correlations were used to investigate if there is any meaningful correlation between these parameters and the $\mathrm{Ni} / \mathrm{Fe}$ abundance ratio. To account for the measurement uncertainties, we use a Monte Carlo method, assuming Gaussian distributions for symmetric uncertainties, skewed Gaussian distributions for asymmetric uncertainties, and a uniform distribution (with a lower limit of $8 \mathrm{M}_{\odot}$ ) for upper limits in the progenitor masses.

We found no significant correlation between the parameters tested above. However, we note that the uncertainties in some parameters are significant. If we do not take into account the uncertainties, we obtain a weak correlation between $\mathrm{Ni} / \mathrm{Fe}$ and $\mathrm{M}_{\max }^{V}$ and progenitor mass. However, these are mainly driven by one object (SN 2012ec).

This null result raises some interesting questions. We did not find a correlation between $\mathrm{M}_{\mathrm{Ni}}$ and $\mathrm{Ni} / \mathrm{Fe}$ abundance ratio, which is expected as one would assume the production of ${ }^{56} \mathrm{Ni}$ to track the production of ${ }^{58} \mathrm{Ni}$ and ${ }^{54} \mathrm{Fe}$ (e.g. J15b). We expected to see an anticorrelation between progenitor mass and $\mathrm{Ni} / \mathrm{Fe}$ abundance 


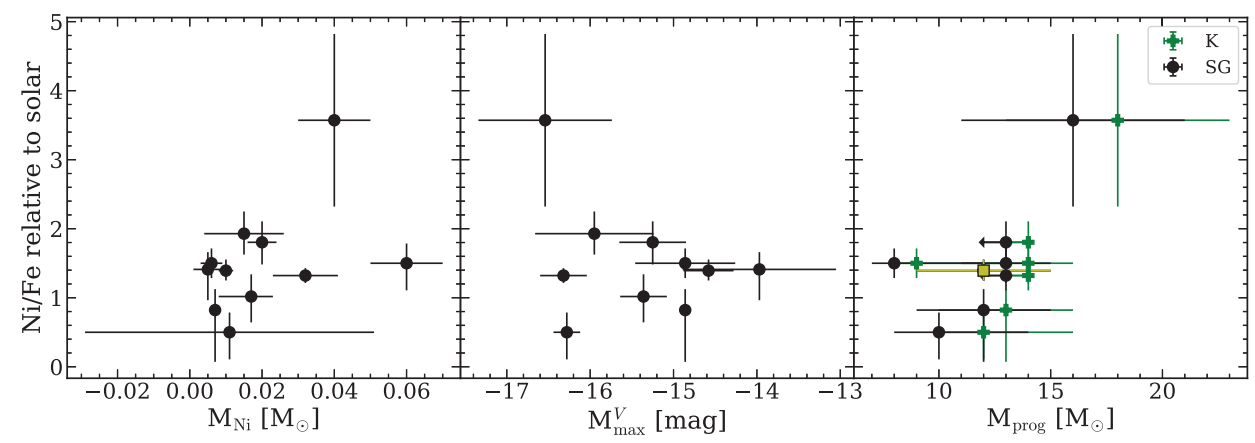

Figure 12. $\mathrm{Ni} / \mathrm{Fe}$ abundance ratio versus $\mathrm{M}_{\mathrm{Ni}}, \mathrm{M}_{\max }^{V}$ and $\mathrm{M}_{\text {prog. }}$. For $\mathrm{M}_{\text {prog }}$, we show two different progenitor models, KEPLER (K) and STARS and Geneva (SG). SN 2016aqf is shown as a yellow square in the subplot with $\mathrm{M}_{\text {prog }}$, for which we assume a value of $12 \pm 3 \mathrm{M}_{\odot}$.

ratio, as theory predicts that lower mass stars have relatively thick silicon shells that more easily encompass the mass cut that separates the ejecta from the compact remnant, ejecting part of their silicon layers, which produces higher $\mathrm{Ni} / \mathrm{Fe}$ abundance ratios. This is supported by the models from Woosley \& Weaver (1995) and Thielemann, Nomoto \& Hashimoto (1996) but not by those from Limongi \& Chieffi (2003), which use thermal bomb explosions instead of pistons, as the former two do (see J15b). Having this in mind, our results either indicate that this anticorrelation can be driven by the exact choice of explosion mechanism (e.g. pistondriven explosions, neutrino mechanism, thermal bomb) and physical parameters (e.g. mass cut, composition, density profile), or that lowmass stars typically do not burn and eject $\mathrm{Si}$ shells but either $\mathrm{O}$ shells or possibly merged O-Si shells (e.g. Collins, Müller \& Heger 2018). This is an important constraint both for pre-SN modelling (shell mergers and convection physics that determines whether these Si shells are thin or thick) and for explosion theory (which matter falls into NS and which is ejected). Finally, we also need to consider the possibility of having primordial $\mathrm{Ni}$ and $\mathrm{Fe}$ contaminating the measured $\mathrm{Ni} / \mathrm{Fe}$ abundance ratio, which could affect our results (as discussed above).

As mentioned in J15b, 1D models tend to burn and eject either $\mathrm{Si}$ shell or $\mathrm{O}$ shell material that gives $\mathrm{Ni} / \mathrm{Fe}$ abundance ratios of $\sim 3$ and $\sim 1$ times solar, respectively. Therefore, there is a clear-cut prediction that we should see a bimodal distribution of this ratio, with relatively few cases where the burning covers both shells. However, the observed distribution of our sample seems to cover the whole $\sim 1-3$ range. This may suggest that the $1 \mathrm{D}$ picture of progenitors is too simplistic. Some of the recent works on multi-D progenitor simulations (e.g. Müller et al. 2016; Collins et al. 2018; Yadav et al. 2020, and references therein) suggest vigorous convection and shell mixing inside the progenitor. If this happens, $\mathrm{Si}$ and $\mathrm{O}$ shells could smear together and burning such a mixture would give rise to $\mathrm{Ni} / \mathrm{Fe}$ abundance ratios covering the observed range depending on the relative masses of the two components.

\section{CONCLUSIONS}

Theoretical modelling has shown that the $\mathrm{Ni} / \mathrm{Fe}$ abundance ratio, which can be estimated from the [Ni II] $\lambda 7378 /[\mathrm{Fe}$ II $] \lambda 7155$ lines ratio, gives an insight of the inner structure of progenitors and explosion mechanism dynamics. To date, very few SNe II have shown these lines in their spectra, most of them been LL SNe II. This could be due to their lower explosion energies (hence, lower expansion velocities), which facilitates the deblending of lines, although these lines have also been found in one SN Ic and SNe Ia.
SN 2016aqf has a similar spectral evolution to other SNe of this faint sub-class and has a bolometric luminosity and expansion velocities that follow the bulk behaviour of LL SNe II. When comparing its nebular spectra to spectral synthesis models to constrain the progenitor mass through the $[\mathrm{OI}] \lambda \lambda 6300,6364$ lines, we find a relatively good agreement with progenitors of 12 (using two model grids) and $15 \mathrm{M}_{\odot}$. However, due to uncertainties (e.g. mixing) in the other models, we cannot exclude lower mass $\left(\sim 9 \mathrm{M}_{\odot}\right)$ progenitors. In addition, we noted that the lack of macroscopic mixing seen in some models produces too much fine structure in the early nebular spectra, which would need to be considered in future modelling. Hence, we conclude that the progenitor of SN 2016aqf had a ZAMS mass of $12 \pm 3 \mathrm{M}_{\odot}$. To further constraint the progenitor mass, a more detailed modelling would be required, although this is outside the scope of this work.

As observed from the theoretical modelling of SNe II progenitors, the presence of $\mathrm{He} \mathrm{I} \lambda 7065$ and $[\mathrm{CI}] \lambda 8727$ in the spectra is linked to the (at least partial) burning of the He shell, which would suggest that SN 2016aqf is a Fe-core SN instead of an ECSN.

SN 2016aqf is a unique case as it has an extended spectral coverage showing the evolution of [Ni II] $\lambda 7378$ and [Fe II] $\lambda 7155$ lines for over $150 \mathrm{~d}$. The ratio between these lines appears to be relatively constant (at $t \gtrsim+170 \mathrm{~d}$ ), which would suggest that one spectrum at a relatively late epoch would be enough to measure this quantity. An optimal epoch range to measure this ratio is $\sim 200-300 \mathrm{~d}$, given that at earlier epochs, the SN can still be in the optically thick phase when the high opacity blocks the contribution from the lines, and at later epochs, the contribution from primordial $\mathrm{Fe}$ and $\mathrm{Ni}$ is more important. This could vary from $\mathrm{SN}$ to $\mathrm{SN}$, so a larger sample with extensive coverage of the [Ni II] $\lambda 7378$ and [Fe II] $\lambda 7155$ lines is required. When comparing to a sample of SNe II (LL and nonLL included) with measured Ni/Fe abundance ratio, the SN 2016 aqf value falls within the middle of the distribution.

We did not find any anticorrelation between ZAMS mass and Ni/Fe abundance ratio as predicted by theory. We believe that this could mean one of two things. On the one hand, as some models predict this anticorrelation, but others do not, this trend could be driven by the choice of explosion mechanism (e.g. piston-driven explosions, neutrino mechanism, thermal bomb) and physical parameters (e.g. mass cut, composition, density profile). On the other hand, this could mean that low-mass stars typically do not burn and eject $\mathrm{Si}$ shells but instead O shells or possibly merged O-Si shells, which would alter the produced $\mathrm{Ni} / \mathrm{Fe}$ abundance ratio. However, one must keep in mind that there is the possibility of having contamination of primordial $\mathrm{Ni}$ and $\mathrm{Fe}$, which can be significant (up to $\sim 40$ per cent) and epoch dependent. 
The current picture of $1 \mathrm{D}$ progenitors may be too simplistic, as higher dimensional effects, like mixing and convection, can play an important role, which could help to reproduce the observed distribution of $\mathrm{Ni} / \mathrm{Fe}$ abundance ratio.

Finally, we note that nebular-phase spectral coverage of SNe II is essential for the study of these objects. While there exist a number of SN II nebular spectra in the literature, additional higher cadence and higher signal-to-noise observations are required to help improve theoretical models.

\section{ACKNOWLEDGEMENTS}

This work is based (in part) on observations collected at the European Organization for Astronomical Research in the Southern Hemisphere under ESO programme 0102.D-0919, and as part of PESSTO, (the Public ESO Spectroscopic Survey for Transient Objects Survey) ESO program 191.D-0935, 197.D-1075. This work makes use of data from Las Cumbres Observatory, the Supernova Key Project, and the Global Supernova Project.

We thank the ASAS-SN collaboration for sharing data of nondetections for this work.

TMB was funded by the CONICYT PFCHA/DOCTORADOBECAS CHILE/2017-72180113. CPG and MS acknowledge support from EU/FP7-ERC grant no. [615929]. SGG acknowledges support by FCT under Project CRISP PTDC/FIS-AST-31546 and UIDB/00099/2020. LG was funded by the European Union's Horizon 2020 research and innovation programme under the Marie Skłodowska-Curie grant agreement no. 839090. This work has been partially supported by the Spanish grant PGC2018-095317-B-C21 within the European Funds for Regional Development (FEDER). MG is supported by the Polish NCN MAESTRO grant 2014/14/A/ST9/00121. MN is supported by a Royal Astronomical Society Research Fellowship. DAH, GH, and CM were supported by NSF grant AST-1313484.

This research has made use of the NASA/IPAC Extragalactic Database (NED), which is operated by the Jet Propulsion Laboratory, California Institute of Technology, under contract with the National Aeronautics. We acknowledge the usage of the HyperLeda database (http://leda.univ-lyon1.fr).

\section{DATA AVAILABILITY}

The photometry and spectra of SN2016aqf are available in the Weizmann Interactive Supernova Data Repository (WISeREP) at https://wiserep.weizmann.ac.il/object/7409. In addition, the photometry of SN 2016aqf is available in this paper.

\section{REFERENCES}

Ambikasaran S., Foreman-Mackey D., Greengard L., Hogg D. W., O’Neil M., 2015, IEEE Trans. Pattern Anal. Mach. Intell., 38, 252

Anderson J. P. et al., 2014, ApJ, 786, 67

Anderson J. P. et al., 2016, A\&A, 589, A110

Arnett D., 1996, Supernovae and Nucleosynthesis: An Investigation of the History of Matter from the big bang to the Present. Princeton University Press, Princeton, NJ

Asplund M., Grevesse N., Sauval A. J., Scott P., 2009, ARA\&A, 47, 481

Barbarino C. et al., 2015, MNRAS, 448, 2312

Bersten M. C., Benvenuto O., Hamuy M., 2011, ApJ, 729, 61

Blondin S. et al., 2006, AJ, 131, 1648

Bose S. et al., 2013, MNRAS, 433, 1871

Brown T. M. et al., 2013, PASP, 125, 1031
Brown J. S. et al., 2016, Astron. Telegram, 8736, 1

Burgh E. B., Nordsieck K. H., Kobulnicky H. A., Williams T. B., O'Donoghue D., Smith M. P., Percival J. W., 2003, Prime Focus Imaging Spectrograph for the Southern African Large Telescope: optical design, p. 1463

Buzzoni B. et al., 1984, Messenger, 38, 9

Cardelli J. A., Clayton G. C., Mathis J. S., 1989, ApJ, 345, 245

Chugai N. N., Utrobin V. P., 2000, A\&A, 354, 557

Collins C., Müller B., Heger A., 2018, MNRAS, 473, 1695

de Jaeger T. et al., 2018, MNRAS, 476, 4592

de Mello D., Benetti S., Massone G., 1997, Int. Astron. Union Circ., 6537, 1

Dessart L. et al., 2014, MNRAS, 440, 1856

Dessart L., Waldman R., Livne E., Hillier D. J., Blondin S., 2013a, MNRAS, 428,3227

Dessart L., Hillier D. J., Waldman R., Livne E., 2013b, MNRAS, 433, 1745

Elmhamdi A. et al., 2003, MNRAS, 338, 939

Firth R. E. et al., 2015, MNRAS, 446, 3895

Fraser M. et al., 2012, ApJ, 759, L13

Gal-Yam A. et al., 2011, ApJ, 736, 159

Gal-Yam A., 2017, Observational and Physical Classification of Supernovae. Springer International Publishing, Cham, p. 195

Galbany L. et al., 2016, AJ, 151, 33

Goldberg J. A., Bildsten L., Paxton B., 2019, ApJ, 879, 3

Guillochon J., Parrent J., Kelley L. Z., Margutti R., 2017, ApJ, 835, 64

Gutiérrez C. P. et al., 2017, ApJ, 850, 89

Gutiérrez C. P. et al., 2018, MNRAS, 479, 3232

Hamuy M., 2003, ApJ, 582, 905

Hamuy M., Pinto P. A., 2002, ApJ, 566, L63

Hamuy M. A., 2001, PhD thesis, The University of Arizona

Harutyunyan A. H. et al., 2008, A\&A, 488, 383

Hosseinzadeh G. et al., 2018, ApJ, 861, 63

Hosseinzadeh G., Yang Y., McCully C., Arcavi I., Howell D. A., Valenti S., 2016, Astron. Telegram, 8748, 1

Jerkstrand A. et al., 2015a, MNRAS, 448, 2482 (J15a)

Jerkstrand A. et al., 2015b, ApJ, 807, 110 (J15b)

Jerkstrand A., Fransson C., Maguire K., Smartt S., Ergon M., Spyromilio J., 2012, A\&A, 546, A28 (J12)

Jerkstrand A., Smartt S. J., Fraser M., Fransson C., Sollerman J., Taddia F., Kotak R., 2014, MNRAS, 439, 3694 (J14)

Jerkstrand A., Ertl T., Janka H. T., Müller E., Sukhbold T., Woosley S. E., 2018, MNRAS, 475, 277 (J18)

Jha S. W., Miszalski B., 2016, Astron. Telegram, 8749, 1

Kasen D., Woosley S. E., 2009, ApJ, 703, 2205

Kennicutt R. C., Evans N. J., 2012, ARA\&A, 50, 531

Kobulnicky H. A., Nordsieck K. H., Burgh E. B., Smith M. P., Percival J. W., Williams T. B., O'Donoghue D., 2003, Prime focus imaging spectrograph for the Southern African large telescope: operational modes. p. 1634

Kozyreva A., Nakar E., Waldman R., 2019, MNRAS, 483, 1211

Kozyreva A., Nakar E., Waldman R., Blinnikov S., Baklanov P., 2020, MNRAS, 494, 3927

Kulkarni S., Kasliwal M. M., 2009, in Kawai N., Mihara T., Kohama M., Suzuki M., eds, Astrophysics with All-Sky X-Ray Observations, p. 312

Kushnir D., 2015, preprint (arXiv:1506.02655)

Lauberts A., Valentijn E. A., 1989, The surface photometry catalogue of the ESO-Uppsala galaxies

Li W. et al., 2011, Nature, 480, 348

Limongi M., Chieffi A., 2003, ApJ, 592, 404

Lisakov S. M., Dessart L., Hillier D. J., Waldman R., Livne E., 2017, MNRAS, 466, 34

Lisakov S. M., Dessart L., Hillier D. J., Waldman R., Livne E., 2018, MNRAS, 473, 3863

Litvinova I. Y., Nadezhin D. K., 1985, Sov. Astron. Lett., 11, 145

Lodders K., 2003, ApJ, 591, 1220

Lyman J. D., Bersier D., James P. A., 2014, MNRAS, 437, 3848

Maeda K. et al., 2007, ApJ, 658, L5

Maeda K., Taubenberger S., Sollerman J., Mazzali P. A., Leloudas G., Nomoto K., Motohara K., 2010, ApJ, 708, 1703

Maguire K. et al., 2010, MNRAS, 404, 981

Maguire K. et al., 2012, MNRAS, 420, 3451 
Makarov D., Prugniel P., Terekhova N., Courtois H., Vauglin I., 2014, A\&A, 570, A13

Marino R. A. et al., 2013, A\&A, 559, A114

Martinez L., Bersten M. C., 2019, A\&A, 629, A124

Maund J. R. et al., 2013, MNRAS, 431, L102

Mazzali P. A., Röpke F. K., Benetti S., Hillebrandt W., 2007, Science, 315, 825

Müller B., Viallet M., Heger A., Janka H.-T., 2016, ApJ, 833, 124

Müller T., Prieto J. L., Pejcha O., Clocchiatti A., 2017, ApJ, 841, 127

Munari U., Zwitter T., 1997, A\&A, 318, 269

Nakaoka T. et al., 2018, ApJ, 859, 78

Papenkova M., Li W., Lotoss/Kait, Schmidt B., Salvo M., Ford A., 2003, IAU Circ., 8143, 2

Park S., Li W., 2004, IAU Circ., 8431, 2

Pastorello A. et al., 2004, MNRAS, 347, 74

Pastorello A. et al., 2006, MNRAS, 370, 1752

Pastorello A. et al., 2009, MNRAS, 394, 2266

Pastorello A., 2012, Memorie della Societa Astronomica Italiana Supplementi, 19, 24

Pejcha O., Prieto J. L., 2015, ApJ, 806, 225

Planck Collaboration XIII, 2016, A\&A, 594, A13

Popov D. V., 1993, ApJ, 414, 712

Poznanski D., Prochaska J. X., Bloom J. S., 2012, MNRAS, 426, 1465

Rasmussen C. E., Williams C. K. I., 2006, Gaussian Processes for Machine Learning

Richmond M. W., Treffers R. R., Filippenko A. V., Paik Y., Leibundgut B., Schulman E., Cox C. V., 1994, AJ, 107, 1022

Riess A. G. et al., 2018, ApJ, 855, 136

Roy R. et al., 2011, ApJ, 736, 76

Sahu D. K., Anupama G. C., Srividya S., Muneer S., 2006, MNRAS, 372, 1315

Schlafly E. F., Finkbeiner D. P., 2011, ApJ, 737, 103

Shappee B. J. et al., 2014, ApJ, 788, 48

Shivvers I. et al., 2017, PASP, 129, 054201

Shussman T., Nakar E., Waldman R., Katz B., 2016, preprint (arXiv:1602.0 2774)

Smartt S. J. et al., 2015, A\&A, 579, A40

Smartt S. J., 2015, PASA, 32, e016

Smartt S. J., Eldridge J. J., Crockett R. M., Maund J. R., 2009, MNRAS, 395, 1409

Spiro S. et al., 2014, MNRAS, 439, 2873

Sukhbold T., Ertl T., Woosley S. E., Brown J. M., Janka H. T., 2016, ApJ, 821,38

Takáts K. et al., 2014, MNRAS, 438, 368

Terry J. N., Paturel G., Ekholm T., 2002, A\&A, 393, 57

Theureau G., Rauzy S., Bottinelli L., Gouguenheim L., 1998, A\&A, 340, 21

Thielemann F.-K., Nomoto K., Hashimoto M.-A., 1996, ApJ, 460, 408

Tomasella L. et al., 2013, MNRAS, 434, 1636

Tomasella L. et al., 2018, MNRAS, 475, 1937

Turatto M. et al., 1998, ApJ, 498, L129

Turatto M., Benetti S., Cappellaro E., 2003, in Hillebrandt W., Leibundgut B., eds, From Twilight to Highlight: The Physics of Supernovae, p. 200,

Utrobin V. P., Chugai N. N., 2009, A\&A, 506, 829

Utrobin V. P., Chugai N. N., Pastorello A., 2007, A\&A, 475, 973

Valenti S. et al., 2014, MNRAS, 438, L101

Valenti S. et al., 2016, MNRAS, 459, 3939

Van Dyk S. D. et al., 2012, AJ, 143, 19

Woosley S. E., Weaver T. A., 1995, ApJS, 101, 181

Woosley S. E., Pinto P. A., Hartmann D., 1989, ApJ, 346, 395

Yadav N., Müller B., Janka H. T., Melson T., Heger A., 2020, ApJ, 890, 94

Yaron O., Gal-Yam A., 2012, PASP, 124, 668

Zampieri L., Pastorello A., Turatto M., Cappellaro E., Benetti S., Altavilla G., Mazzali P., Hamuy M., 2003, MNRAS, 338, 711

Zhang J. et al., 2014, ApJ, 797, 5

\section{APPENDIX A: NICKEL MASS ESTIMATION}

In the literature, there are various methods to estimate the ${ }^{56} \mathrm{Ni}$ mass. These are as follows.

Arnett (1996) gives the following relation using SN 1987A as comparison:

$M_{\mathrm{Ni}}=0.075 \times \frac{L_{\mathrm{SN}}}{L_{87 \mathrm{~A}}} \mathrm{M}_{\odot}$

By using the bolometric light curve calculated in Section 3.4, interpolating with Gaussian Processes to obtain the luminosity at $200 \mathrm{~d}$ after the explosion, we obtain $M_{\mathrm{Ni}}=0.008 \pm 0.001 \mathrm{M}_{\odot}$.

Hamuy (2003) formed a relation between the bolometric luminosity of the exponential decay tail and the ${ }^{56} \mathrm{Ni}$ mass. The bolometric luminosity is then given by:

$$
\begin{aligned}
& \log _{10} L_{\text {tail }} \\
& \quad=\frac{-\left[m_{V, \text { tail }}-A_{\mathrm{MW}}(V)-A_{\text {Host }}(V)+\mathrm{BC}\right]+5 \log _{10}(D)-8.14}{2.5}
\end{aligned}
$$

where $L_{\text {tail }}$ is the tail luminosity in $\operatorname{erg~s}^{-1}$ at $200 \mathrm{~d}$ after the explosion, $D$ is the distance in $\mathrm{cm}, \mathrm{BC}$ is a bolometric correction that permits one to transform $V$ magnitudes into bolometric magnitudes, and the additive constant provides the conversion from Vega magnitudes into cgs units. From SN 1987A and SN 1999em, Hamuy (2001) found that $\mathrm{BC}=0.26 \pm 0.06$. Using the relation found by Hamuy (2003), the nickel mass is obtained as follows:

$M_{\mathrm{Ni}}=\left(7.866 \times 10^{-44}\right) L_{\text {tail }} \exp \left[\frac{\left(t_{\text {tail }}-t_{0}\right) /(1+z)-6.1}{111.26}\right] \mathrm{M}_{\odot}$

from which we obtain $M_{\mathrm{Ni}}=0.011 \pm 0.003 \mathrm{M}_{\odot}$.

Maguire et al. (2012) found a relation between the nickel mass and the $\mathrm{H} \alpha$ FWHM given by

$M_{\mathrm{Ni}}=A \times 10^{B \times \mathrm{FWHM}_{\mathrm{corr}}} \mathrm{M}_{\odot}$

where $B=0.0233 \pm 0.0041, A=1.81_{-0.68}^{+1.05} \times 10^{-3}$, and $\mathrm{FWHM}_{\text {corr }}$

is the FWHM of $\mathrm{H}_{\alpha}$, corrected by the spectral resolution of the instrument, during the nebular phase $(\sim 350-550 \mathrm{~d})$. From this relation, using the FWHM of $\mathrm{H} \alpha$ from the spectrum at $+348 \mathrm{~d}$, we obtain $M_{\mathrm{Ni}}=0.014_{-0.007}^{+0.009} \mathrm{M}_{\odot}$, where we used $\mathrm{FWHM}_{\text {inst }}=21.2$ $\AA$, taken from grism \#13 in EFOSC2 (as given in the ESO website).

$\mathrm{J} 12$ also gives a relation to estimate the nickel mass from the early exponential-decay tail, assuming full trapping, that the deposited energy is instantaneously re-emitted and that no other energy source has any influence, i.e.

${ }_{56}{ }_{\mathrm{Co}}(t)=9.92 \times 10^{41} \frac{M_{\mathrm{Ni}}}{0.07 M_{\odot}}\left(e^{-t / 111.4 d}-e^{-t / 8.8 d}\right) \mathrm{erg} \mathrm{s}^{-1}$

from which we obtain $M_{\mathrm{Ni}}=0.007 \pm 0.001 \mathrm{M}_{\odot}$.

\section{APPENDIX B: $V$-BAND COMPARISON}

Given that the SN was not visible for a period of time, we do not have observations of the transition from the plateau phase to the nebular phase. To estimate the duration of the plateau, we therefore compared the $V$-band light curve of SN 2016aqf with other LL SNe II in our sample. We found that the $V$ band of SN 2003fb has a similar shape (see Fig. B1), if normalized by the luminosity at $50 \mathrm{~d}$ after the explosion. For this reason, we decided to use the plateau duration of SN 2003fb (adding its uncertainty in quadrature) for SN 2016aqf. 


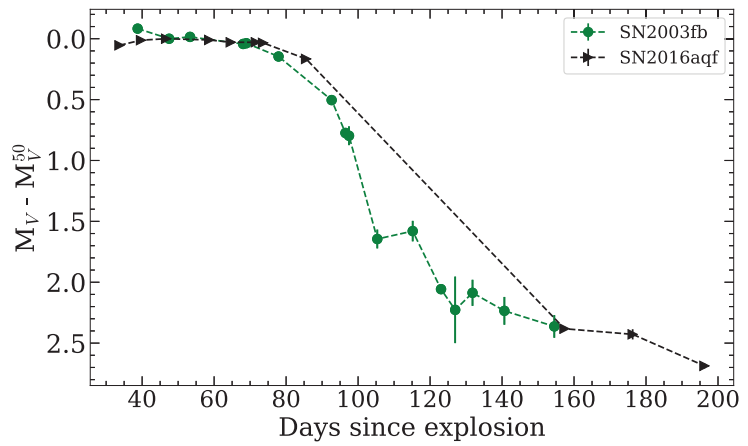

Figure B1. $V$-band comparison between the light curves of SN 2016aqf and $\mathrm{SN} 2013 \mathrm{fb}$. The $y$-axis is $V$-band absolute magnitude minus $V$-band absolute magnitude at $+50 \mathrm{~d}$. The evolution of both light curves is very similar.

${ }^{1}$ School of Physics and Astronomy, University of Southampton, Southampton, Hampshire, SO17 1BJ, UK

${ }^{2}$ The Oskar Klein Centre, Department of Astronomy, Stockholm University, AlbaNova, SE-106 91 Stockholm, Sweden

${ }^{3}$ Max-Planck Institut für Astrophysik, Karl-Schwarzschild Str 1, D-85748 Garching, Germany

${ }^{4}$ European Southern Observatory, Alonso de Córdova 3107, Casilla 19, Santiago, Chile

${ }^{5}$ CENTRA/COSTAR, Instituto Superior Técnico, Universidade de Lisboa, Av. Rovisco Pais 1, P-1049-001 Lisboa, Portugal

${ }^{6}$ The School of Physics and Astronomy, Tel Aviv University, Tel Aviv 69978, Israel
${ }^{7}$ CIFAR Azrieli Global Scholars program, CIFAR, Toronto, Canada

${ }^{8}$ Las Cumbres Observatory, Goleta, CA 93117, USA

${ }^{9}$ Department of Physics, University of California, Santa Barbara, CA 93106, USA

${ }^{10}$ Departamento de Física Teórica y del Cosmos, Universidad deGranada, E-18071 Granada, Spain

${ }^{11}$ Department of Particle Physics and Astrophysics, Weizmann Institute of Science, Rehovot 76100, Israel

${ }^{12}$ Astronomical Observatory, University of Warsaw, Al. Ujazdowskie 4, PL00-478 Warszawa, Poland

${ }^{13}$ Center for Astrophysics | Harvard \& Smithsonian, 60 Garden Street, Cambridge, MA 02138, USA

${ }^{14}$ School of Physics \& Astronomy, Cardiff University, Queens Buildings, The Parade, Cardiff CF24 3AA, UK

${ }^{15}$ Department of Physics and Astronomy, University of Turku, Vesilinnantie 5, FI-20014 Turku, Finland

${ }^{16}$ Birmingham Institute for Gravitational Wave Astronomy and School of Physics and Astronomy, University of Birmingham, Birmingham B15 2TT, UK

${ }^{17}$ Institute for Astronomy, University of Edinburgh, Royal Observatory, Blackford Hill EH9 3HJ, UK

${ }^{18}$ Astrophysics Research Centre, School of Mathematics and Physics, Queens University Belfast, Belfast BT7 1NN, UK

${ }^{19}$ Department of Physics, University of California, 1 Shields Avenue, Davis, CA 95616, USA

This paper has been typeset from a $\mathrm{T}_{\mathrm{E}} \mathrm{X} / \mathrm{L} \mathrm{T} \mathrm{E} \mathrm{X}$ file prepared by the author. 